FUNCTIONAL SCREENING OF ZEBRAFISH CYTOCHROME P450 1 BY CE 


\title{
FUNCTIONAL SCREENING OF CYTOCHROME P450 ACTIVITY AND UNCOUPLING BY CAPILLARY ELECTROPHORESIS
}

\author{
By
}

JAMES G HARSKAMP, B.Sc.

A Thesis Submitted to the School of Graduate Studies in Partial Fulfilment of the Requirements for the Degree Master of Science 
McMaster University MASTER OF SCIENCE (2011) Hamilton, Ontario (Biology)

TITLE: Functional Screening of Cytochrome P450 Activity and Uncoupling by Capillary Electrophoresis

AUTHOR: James G. Harskamp, B.Sc. (Redeemer University College)

SUPERVISOR: Dr. J.Y. Wilson

NUMBER OF PAGES: ix, 74 


\begin{abstract}
:
Cytochrome P450s are a super-family of heme containing proteins that are found in all domains of life and are involved in the synthesis and breakdown of steroids, xenobiotics, and pharmaceuticals. Using five heterologously expressed zebrafish (Danio rerio) CYP1s, an assay was developed for CYP activity in order to monitor the consumption of the cofactor NADPH, providing a label-free screening tool to determine function of novel CYP genes. Using wellestablished fluorogenic substrates, NADPH and $\mathrm{NADP}^{+}$were separated by capillary electrophoresis (CE) from stopped CYP1 reactions and measured with UV absorbance detection as a surrogate to assess the rate of substrate metabolism. Product formation was confirmed by fluorometric detection of metabolites, giving rates of enzyme activity which could be compared to the rates of cofactor turn-over measured by CE. 17 $\beta$-estradiol, four alkoxyresorufin and two coumarin based synthetic fluorogenic CYP substrates were screened for activity with recombinant zebrafish CYP1A, 1B1, 1C2, 1C2 and 1D1. Cofactor consumption was generally much larger than product formation for the majority of substrates and CYP1 isoforms, suggesting that the majority of metabolic events were uncoupled. Large uncoupling was seen in CYP1 when metabolizing estradiol, showing that endogenous compounds can also show severe uncoupling. Reactive oxygen species (ROS), a product of uncoupled events, were detected with 2,7dichorofluorescein. Attempts for concomitant detection of ROS production and cofactor consumption with CE-UV detection were investigated, however, detection limits for 2,7dichlorofluorescein were not adequate for detection of hydrogen peroxide production from CYP1 mediated reactions. Future work will be required to develop a single assay to quantitatively measure CYP activity by CE for functional determination of CYPs with unknown function.
\end{abstract}




\section{Acknowledgements:}

I would like to thank my supervisors Dr. Joanna Wilson and Dr. Philip Britz-McKibbin for all their support throughout my experiments and writing. The opportunity to do graduate studies in both Biology and Chemistry was one I would not want to pass up. I would also like to thank the Wilson and Britz-McKibbin lab groups members for all their support, coffee breaks, and tips. It has been a pleasure to work with such enjoyable people.

I would also like to thank my family. Thank you to Doug and Vanessa and Brian, who opened their spaces to me often for me to do my writing. Thank you to mom and dad Sikkema who regularly took interest in what my project was despite not understanding any of it. And most of all, thank you to my wife, Rebecca, who gave me support throughout my two years here. Without your encouragement and love I would not have enjoyed this project nearly as much. 


\section{Table of Contents}

\section{Chapter 1: General Introduction}

1.1 Cytochrome P450 enzymes................................................................................................ 1

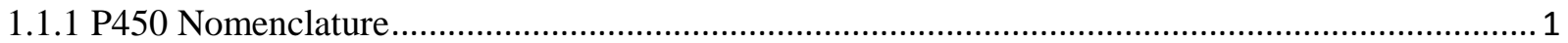

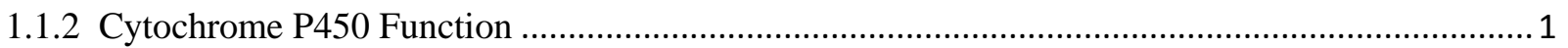

1.1.3 Cytochrome P450 Reductase and the CYP Catalytic Cycle .................................................... 2

1.1.4 Coupling efficiency and uncoupling in the P450 catalytic cycle ............................................... 4

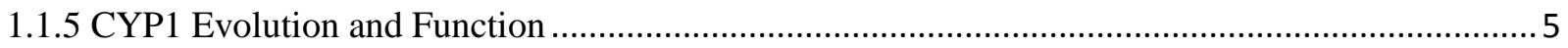

1.1.6 Measurement of CYP1 Activity and ROS production ............................................................ 7

1.2 Capillary Electrophoresis $(\mathrm{CE})$...................................................................................................... 10

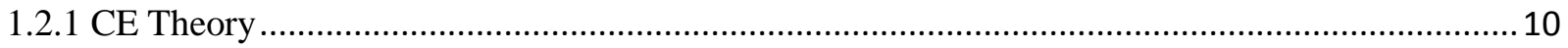

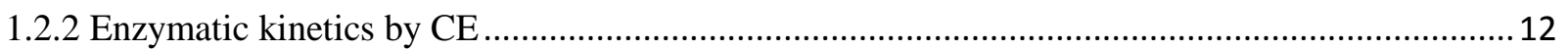

1.3 Research Goals and Questions....................................................................................................... 14

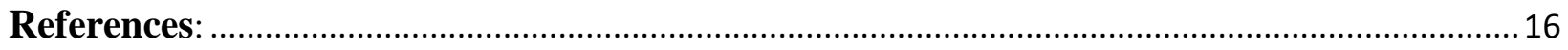

Chapter 2: Functional Screening of Cytochrome P450 Activity and Uncoupling by Capillary Electrophoresis

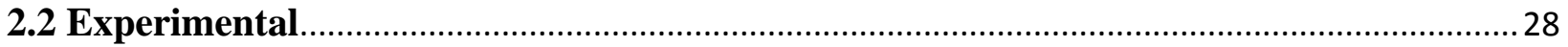

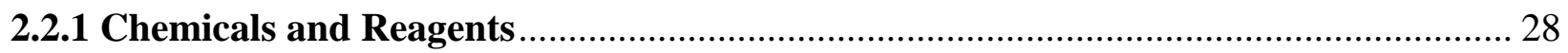

2.2.2 Preparation of Heterologously Expressed Recombinant Zebrafish CYP1s ............... 28

2.2.3 CYP1 Activity Measurements Based on Product Turn-over Rates by Fluorescence.. 29

2.2.4 CYP1 Activity Measurements Based on Rate of NADP+ Production by CE............. 30

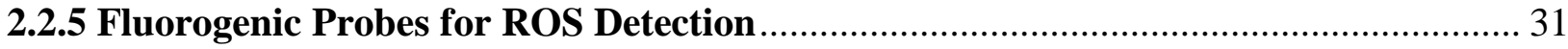

Chapter 3: Dichlorofluorescein product separation with CE-UV

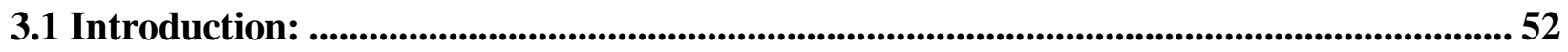

3.2 Materials and Methods: ............................................................................................................... 53

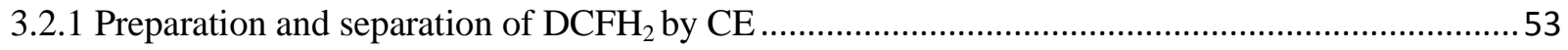

3.2.2 CYP1A Membrane preparation and CYP reactions................................................................. 54

3.2.3 Separation of Samples by CE-UV .................................................................................... 54 
3.3 Results and Discussion

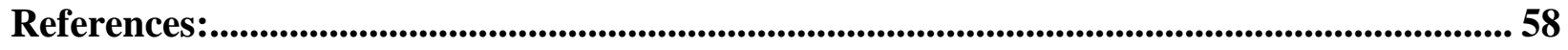

\section{Chapter 4: General Discussion}

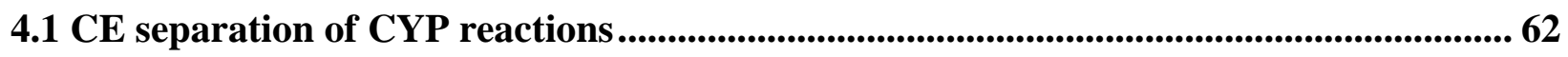

4.2 ROS detection and separation of DCF and HE by CE................................................ 64

4.3 Uncoupling of CYP reactions and inference of CYP function......................................... 67

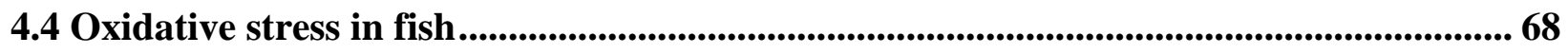

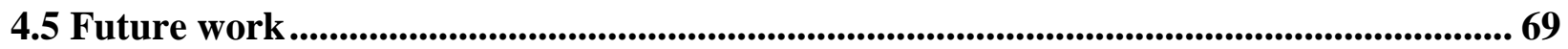

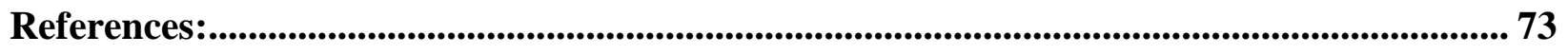




\section{List of Tables:}

Chapter 2: Functional Screening of Cytochrome P450 Activity and Uncoupling by Capillary Electrophoresis

Table 1. Comparison of the catalytic activity of zebrafish CYP1s1 for six different fluorogenic substrates as measured by fluorescence and CE, respectively.......................................... 41

Table S1. Structures and properties of synthetic fluorogenic probes used for assessment of CYP1 activity and coupling efficiency

Table S2. CE-UV detection assay parameters for NADPH and $\mathrm{NADP}^{+}$quantification............. 47 


\section{List of Figures}

\section{Chapter 1: General Introduction}

Figure 1.1: Binding of substrates near the heme of CYP enzymes. ......................................20

Figure 1.2: The general apparatus for capillary electrophoresis $(\mathrm{CE})$. . .......................21

Figure 1.3: The migration order of ions and neutral compounds in a fused-silica capillary under basic conditions

\section{Chapter 2: Functional Screening of Cytochrome P450 Activity and Uncoupling by Capillary Electrophoresis}

Figure 1: Assessment of zebrafish CYP1A activity for O-dealkylation of a homologous series of resorufin substrates (inset) using $\mathrm{CE}$ based on the rate of $\mathrm{NADP}^{+}$production relative to reductase blank

Figure 2: Substrate-dependence on apparent coupling efficiency for recombinant CYP1 isoforms

Figure 3: Activity of zebrafish CYP1 isoforms for $17 \beta$-estradiol .................................43

Figure S1: Multi-step redox cycle associated with enzyme-catalyzed oxidation ...........44

Figure S2: Scheme of rapid separation of cofactor $\left(\mathrm{NADPH} / \mathrm{NADP}^{+}\right.$) turn-over rates in a CYP mediated reaction by pressure-assisted CE under reverse polarity ........................46

Figure S3: Comparison of zebrafish CYP1 activity for $O$-dealkylation of 7ethoxyresorufin

Figure S4: Impact of ascorbic acid palmitate and ascorbic acid as hydrogen peroxide scavengers in CYP1 mediated reactions with $17 \beta$-estradiol

Figure S5: Comparison of extent of CYP1 uncoupling induced by PCB 77 .50

\section{Chapter 3: Dichlorofluorescein product separation with CE-UV}

Figure A1: Reactions needed for the production of the fluorescent probe dichlorofluorescein .59

Figure A2: Separation of 2,7-dichlorodihydrofluorescein diacetate products with CE ...60 


\section{Abbreviations:}

7-BR: 7-benzyloxyresorufin

7-ER: 7-ethyoxyresorufin

7-MR: 7-methoxyresorufin

7-PR: 7-pentoxyresorufin

AROD: alkoxyresorufin O-dealkylase

BFC: 7-benzyloxy-4-trifluoromethylcoumarin

BGE: background electrolyte

BROD: 7-benxyloxyresorufin O-dealkylase

CE: capillary electrophoresis

CEC: 3-cyano-7-ethoxycoumarin

CYP: cytochrome P450

DCFH2-DA: 2,7-dichlorodihydrofluorescein diacetate

DCFH2: 2,7-dichlorodihydrofluorescein,

DCF: 2,7-dichlorofluorescein

$\mathbf{E}_{\mathbf{2}}$ : $17 \beta$-estradiol

EMMA: electrophoretically mediated microanalysis

EOF: electoosmotic flow

EROD: 7-ethoxyresorufin O- dealkylase

$\mathbf{H}_{2} \mathbf{E}$ : dihydroethidium

HE: hydroethidium

MROD: 7-methoxyresorufin O- dealkylase

NADPH: reduced nicotinamide adenine dinucleotide phosphate

NADP $^{+}$: nicotinamide adenine dinucleotide phosphate

P450 (or CYP): cytochrome P450

PAH: polyaromatic hydrocarbon

PCB: polychlorinated biphenyl

PROD: 7-pentoxyresorufin O- dealkylase

ROS: reactive oxygen species 


\section{Chapter 1:}

\section{General Introduction}

\subsection{Cytochrome P450 enzymes}

\subsubsection{P450 Nomenclature}

Cytochrome P450's enzymes (CYPs) are a large family of hemoproteins found in all domains of life. With the advancement of genomics and other disciplines, many previously unknown CYP genes are being identified and categorized (Denisov et al., 2005; Nelson et al., 1996). A nomenclature system was developed in order to consistently name genes based on amino acid identity at the time of discovery, such that genes are placed in the same family and subfamily when they have $>40 \%$ and $>55 \%$ primary amino acid sequence overlap, respectively; individual genes are given numbers in order of discovery (Nelson et al., 1996). For example, the enzyme aromatase is encoded by the CYP19A1 gene, the $1^{\text {st }}$ gene in the ' $A$ ' subfamily of the CYP19 family.

\subsubsection{Cytochrome P450 Function}

CYP proteins play critical roles in the oxidative metabolism of endogenous compounds, as well as xenobiotic (exogenous) compounds, including pharmaceuticals and environmental toxins. Some isoforms have very specific functions with a limited number of substrates, such as aromatase (CYP19A1) which transforms androgens into estrogens (Diotel et al., 2010). Other CYPs have much broader specificity with numerous substrates such as CYP3A4, which is able to metabolize approximately $50 \%$ of all pharmaceuticals in mammals (Zhang et al., 2006). 
Cytochrome P450 families 1, 2, and 3 are predominantly expressed in the liver and are known to be the main catalysts in xenobiotic metabolism. Many CYPs show very flexible active sites which can accommodate various substrates for access to the heme of the protein (Guengerich, 2001; Martignoni et al., 2006b). In general, CYP substrates are hydrophobic and become more soluble when metabolised by adding oxygen into the molecule (Denisov et al., 2005). Common oxidative transformations catalyzed by CYPs include, carbon hydroxylation, heteroatom oxidation, dealkylation, and epoxidation, however there are many more reactions possible with an extensive review of transformations given by Guengerich (2001).

Mammalian CYP isoforms in the same subfamily display significant variations in their activity and regiospecificity for substrate oxidation (Martignoni et al., 2006a; Martignoni et al., 2006b). Furthermore, comparisons of homologous CYP isoforms in mammalian and nonmammalian vertebrate species have shown large disparity in catalytic function (Scornaienchi et al.; Scornaienchi et al., 2010a). CYP homologs may be found in certain species as pseudogenes and yet be functional in evolutionary similar species, such as CYP1D1 which is functional in Cynomolgus monkeys and a pseudogene in humans (Uno et al., 2011). While gene sequences may give insight into the putative function of a particular CYP, predictions are not always correct and may provide little information on its biological role in metabolism.

\subsubsection{Cytochrome P450 Reductase and the CYP Catalytic Cycle}

The CYP catalytic cycle is a multistep process where electrons are donated from the cofactor NADPH to the heme of the CYP in order for oxygen addition to occur on the substrate based on the net equation: 


$$
\mathrm{RH}+\mathrm{NADPH}+\mathrm{H}^{+}+\mathrm{O}_{2} \rightarrow \mathrm{ROH}+\mathrm{NADP}^{+}+\mathrm{H}_{2} \mathrm{O}
$$

where $\mathrm{RH}$ is the substrate and $\mathrm{ROH}$ is the product.

The initial steps of the catalytic cycles are the binding of the substrate in the active site of the CYP close to the ferric $\left(\mathrm{Fe}^{3+}\right)$ heme centre followed by donation of electrons from NADPH through the separate electron transport protein, cytochrome $\mathrm{P} 450$ reductase (CPR;Guengerich, 2001;Figure S1, Chapter 2). The CPR is a $78 \mathrm{kDa}$ flavoprotein with the electron accepting flavin adenine dinucleotide (FAD) and flavin monocucleotide (FMN) moieties which allows for electron flow to occur one at a time toward the heme (Hamdane et al., 2009). The electron transfer changes the charge on the heme from $\mathrm{Fe}^{3+}$ to the ferrous state $\left(\mathrm{Fe}^{2+}\right)$ which can bind a molecule of $\mathrm{O}_{2}$ to form a Fe${ }^{2+}-\mathrm{O}_{2}$ complex (Guengerich, 2001). Another electron donation step occurs from NADPH via $\mathrm{CPR}$ or the cytochrome $b_{5}$ protein, a ubiquitous electron transporter often found in microsomal and mitochondrial systems. After the second electron transfer, a proton is added and the $\mathrm{O}-\mathrm{O}$ bond is cleaved to form $\mathrm{H}_{2} \mathrm{O}$ and $\mathrm{Fe}(\mathrm{OH})^{2+}$ complex which can selectively add $\mathrm{OH}$ to the docked substrate to generate the oxidized product (Denisov et al., 2005). The iron then returns to its initial $\mathrm{Fe}^{3+}$ state, where it can repeat the cycle for additional substrate metabolism. The catalytic cycle for CYP oxidative metabolism is depicted in Chapter 2, Figure $\mathrm{S} 1$. Cytochrome $\mathrm{b}_{5}$ is thermodynamically unfavoured compared to CPR for the initial electron donation to the heme, however is able to donate for the second electron and can potentially increase the rate of substrate metabolism by CYP (Guengerich, 2001; Scornaienchi et al., 2010a; Sue Masters and Marohnic, 2006). 


\subsubsection{Coupling efficiency and uncoupling in the $\mathbf{P 4 5 0}$ catalytic cycle}

CYP reactions are not always efficient and electron transfer may be uncoupled to substrate binding, causing the consumption of NADPH without product formation. During uncoupled reactions, electrons are donated from NADPH to form superoxide $\left(\mathrm{O}_{2}{ }^{-}\right)$, hydrogen peroxide $\left(\mathrm{H}_{2} \mathrm{O}_{2}\right)$, or water (Gorsky et al., 1984). CYP isoforms that are highly regioselective or stereoselective in substrate metabolism, such as CYP19A1, are relatively efficient in cofactor consumption (Denisov et al., 2005). These typically include CYPs responsible for the production of important biological signalling molecules, such as steroids; reactions that are oxidized by CYPs outside families 1-3 in vertebrate species. Other CYPs, such as CYP3A4, can metabolise a large number of structurally different substrates, and are less specific in substrate binding (Ansede and Thakker, 2004). Overall, CYPs involved in metabolism of xenobiotics show a greater tendency for catalytic uncoupling due to flexibility of the active site (Denisov et al., 2005). While some uncoupling can be due to high levels of NADPH used in vitro where additional NADPH only adds to peroxide formation (Degregorio et al., 2011), other uncoupling events are attributed to successful docking of substrate within the active site of the protein but too far from the heme centre to elicit efficient metabolism (Prasad et al., 2007). In its resting state, the iron heme of the CYP is $\mathrm{Fe}^{3+}$ with a water molecule acting as a ligand to the heme. If a substrate is able to enter the active site in close proximity to the heme, the water molecule is displaced, causing the heme to go from a low-spin state to a high-spin state, which is thermodynamically more favoured to accept an electron from CPR and reduce the heme to $\mathrm{Fe}^{2+}$ (Sligar, 1976). In silico work with theoretical docking of substrates into mammalian and teleost CYP1As showed the rate of substrate oxidation was dependant on substrate positioning within 4 $\AA$ from the heme centre for the substrate benzo[a]pyrene (Prasad et al., 2007; Figure 1.1). The 
substrate's ability to bind to the active site with an appropriate orientation is a key step in the CYP catalytic cycle, which can later become uncoupled in three different pathways resulting in formation of reactive oxygen species (ROS).

The first potential product during uncoupled reactions is superoxide formation from the dissociation of the relatively unstable $\mathrm{Fe}^{2+} \cdot \mathrm{O}_{2}$ complex formed after $\mathrm{O}_{2}$ binds to the CYPsubstrate complex (Guengerich, 2001). $\mathrm{O}_{2}{ }^{-\cdots}$ is rapidly reduced in vivo to $\mathrm{H}_{2} \mathrm{O}_{2}$ via superoxide dismutase. The second possible product during uncoupling is hydrogen peroxide $\left(\mathrm{H}_{2} \mathrm{O}_{2}\right)$, formed from the dissociation of the $\mathrm{Fe}-\mathrm{OOH}$ complex after the addition of the second electron (Denisov et al., 2005). Interestingly, the reverse reaction, called the peroxide shunt, can occur where $\mathrm{H}_{2} \mathrm{O}_{2}$ can be substituted for an oxygen and a reducing equivalent to continue the cycle (Ansede and Thakker, 2004). Finally, two water molecules can be produced by using two reducing equivalents, to restart the cycle without product formation (Gorsky et al., 1984; Zhang et al., 2008b). The uncoupled pathways which produce ROS are shown in Chapter 2, Figure S2. The implication of formation of ROS from biological systems has been well documented and can impact antioxidant defence mechanisms and cell phenotype via redox signalling (Groeger et al., 2009; Winterbourn and Hampton, 2008). Recently, the role of CPR in ROS formation and depletion has suggested an alternative heme-independent mechanism of uncoupling (Manoj et al., 2010), adding to the need for a better understanding of the implications of inefficient CYP metabolism in organisms.

\subsubsection{CYP1 Evolution and Function}

The CYP1 family contains 4 known subfamilies in vertebrate species (CYP1A, CYP1B, CYP1C and CYP1D) but only CYP1A and CYP1B enzymes appear to be present in all 
vertebrates and have been the most studied. Mammalian species contain only the CYP1A and 1B subfamilies, and the CYP1C subfamily has only been identified in non-mammilian vertebrates (Goldstone et al., 2007). CYP1D1 was first identified in fish (Goldstone et al., 2009), and recently has been found in cynomolgus monkey (Uno et al., 2011).

Three CYP1 genes, CYP1A1, CYP1A2, and CYP1B1 are found in humans. CYP1A1 is known to metabolise both xenobiotic compounds such as PAHs and PCBs, and can also metabolise endogenous compounds, such as estrogens (Nebert et al., 2004). CYP1A2 is known to metabolize caffeine (Uno et al., 2011) and aromatic amines (Nebert et al., 2004). Both CYP1A1 and CYP1A2 can be classified by the metabolism of the synthetic substrates 7ethoxyresorufin and 7-methoxyresorufin, respectively (Huang and Szklarz, 2010). CYP1B1 is the only isoform of the subfamily, and is known to be involved in the metabolism of estradiol, PAHs, arylamines, and other carcinogens (Nebert et al., 2004).

Non-mammalian vertebrates exhibit different CYP1s than those present in mammals. Most notably, the CYP1C subfamily is not present in mammals but has been identified in frogs (Jonsson et al., 2011), birds (Goldstone et al., 2007), and fish (Zanette et al., 2009). There is only one isoform in the CYP1A family in fish and this gene (CYP1A) has the rare nomenclature such that the CYP has not been provided a gene number. The function of teleost CYP1A appears to be closer to CYP1A1 in mammals, than CYP1A2, and it is able to metabolise both 7ethoxyresorufin and 7-methoxyresorufin (Scornaienchi et al., 2010b). Teleost CYP1A appears to have higher activity for estradiol than CYP1B1 but CYP1B1 can metabolize traditional mammalian CYP1 substrates including 7-ethoxyresorufin (Scornaienchi et al., 2010a). There were differences between metabolism of CYP1C1 and CYP1C2 with regards to estrogen and PAH metabolism, yet there appears to be great overlap in function of CYP1A, CYP1B1 and both 
CYP1C genes in zebrafish (Scornaienchi et al., 2010a; Scornaienchi et al., 2010b). Zebrafish CYP1D1 showed minimal metabolism for alkoxyresorufin $-O-$ dealkylation (AROD) substrates, and showed little product formation for benzo[a]pyrene or estradiol metabolism, leaving CYP1D1 as the only expressed isoform for which a well metabolised substrate has yet to be identified, suggesting a very different function from other CYP1 genes in spite of the shared evolutionary homology and sequence similarity (Scornaienchi et al., 2010b). A functional CYP1D1 has been found in several mammalian genomes, yet it is a pseudogene in several mammalian species including human, indicating that this isoform is not restricted to nonmammalian vertebrates as first thought (Kawai et al., 2010). The identification of CYP1C and CYP1D genes through genomics highlights that the number of CYPs being discovered through genomics is increasing, providing a large number of CYP sequences that have not been functionally characterized. As many CYPs contribute to the health and cellular defence of the organisms, a method to measure and identify CYP function is needed to ascertain the function of novel CYPs.

\subsubsection{Measurement of CYP1 Activity and ROS production}

The most common way to measure CYP activity is to monitor the rate of substrate consumption, and/or the metabolites generated. This is often done with separation techniques, such as gas chromatography (GC), liquid chromatography (LC), or capillary electrophoresis (CE) coupled with a detector such as UV absorbance or mass spectrometry (MS) (Ansede and Thakker, 2004; Scornaienchi et al., 2010a; Zhang et al., 2008b). However, with each substrate and metabolite of interest, a new method selecting for compounds is needed. This extra step of method development for each substrate of interest slows down the analysis and increases the cost 
needed for CYP screening. Alternatively, high-throughput screening of CYP activity can also be performed using fluorescence technology based on fluorescent product formation from a synthetic fluorogenic substrate. A series of fluorogenic substrates with high specificity for particular mammalian CYP isoforms are then used to probe the activity of test analytes based on fluorescence inhibition, such as drugs and environmental toxins (Smith and Wilson, 2010) Typically, the substrates have been designed for mammalian CYP isoforms belonging to the CYP1A, CYP2C, CYP2D, or CYP3A subfamilies (Lewis et al., 2004; Renwick et al., 2000) . However, fluorescence based screening methods suffer from potential spectral/quenching interferences while also requiring that a substrate or products for a specific CYP isoform to be known in advance. Unfortunately, an understanding of the function might not be known if the only information available is genomic since conservation of function across homologous CYP isoforms cannot be presumed. In fact, the available substrates have been carefully characterized only for mammalian CYP isoforms and the applicability of these substrates as selective markers of non-mammalian CYP isoform activity has not been as widely tested. In fish systems, these synthetic probes do not appear to have the same selectivity as mammalian systems (Smith and Wilson 2010).

Other possible ways to measure CYP activity include monitoring other reagents or products in the reaction, namely NADPH/NADP ${ }^{+}, \mathrm{O}_{2}$, or ROS formation. A common way to measure cofactor consumption is to monitor depletion of NADPH at $340 \mathrm{~nm}$ using UV absorbance, giving a general rate of the reaction (Degregorio et al., 2011; Gorsky et al., 1984; Schlezinger et al., 1999). More recently, CE - UV has been used to monitor NADPH and $\mathrm{NADP}^{+}$consumption in CYP reactions in order to determine rate of pharmaceutical metabolism (Zhang et al., 2008b; Zhang et al., 2006) However, these methods have often been criticized for having inadequate 
blanks from homogenized tissue and cannot correct for individual CYP activity or CPR activity in formation of ROS, leading to large errors in measurement (Ansede and Thakker, 2004). The measurement of $\mathrm{O}_{2}$ consumption in a CYP mediated reaction has been used previously, however some studies have showed little success due to the low sensitivity of $\mathrm{O}_{2}$ monitoring (Dike et al., 2000).

Finally, ROS measurements have been attempted in order to understand cofactor consumption and uncoupling. The most common methods are to use probes that produce a fluorescent product when reacted with ROS. The most common probes are dihydroethidium (HE) and dichlorofluorescein (DCF) which are selective for superoxide and hydrogen peroxide, respectively (Afri et al., 2004; Xu and Arriaga, 2009) . Some groups have used these probes to investigate ROS in CYP reactions (Ramadass et al., 2003; Schlezinger et al., 2006; Viswanathan et al., 2003), to investigate toxin-induced oxidative stress and CYP inhibition. There has been controversy with each probe as the mechanisms of product formation is not fully known, and appear less selective for specific a ROS species than previously thought (Chen et al., 2010; Papapostolou et al., 2004; Zhao et al., 2003). Other groups have used colorometric assays to measure CYP-induced ROS production as a means to correct for excessive cofactor consumption (Degregorio et al., 2011; Huang and Szklarz, 2010), however these methods involve complicated sample handling, low sample throughput and lower sensitivity than fluorescence methods. 


\subsection{Capillary Electrophoresis (CE)}

\subsubsection{CE Theory}

Capillary electrophoresis (CE) is a technique that is able to separate complex mixtures based on the charges of the individual molecules. The separations take place in open tubular fused-silica capillaries with an inner diameter less than $100 \mu \mathrm{m}$, which greatly increases separation efficiency, peak capacity and heat dissipation. Two unique electrokinetic factors drive the separation process in CE, namely the electrophoretic mobility $\left(\mu_{\mathrm{ep}}\right)$ and the electroosmotic flow (EOF).

The $\mu_{\mathrm{ep}}$ is dependent on the properties of the analyte measured in the separation and is described by the ion velocity (v) per unit of electric field strength (E). The mobility of the ion can also be described as the ratio of effective charge $\left(\mathrm{z}_{\mathrm{eff}}\right)$ to hydrated radius $\left(\mathrm{R}_{\text {hyd }}\right)$ of the analyte which is in a background electrolyte (BGE) of a particular viscocity $(\eta)$, giving:

$$
\mu_{e p}=\frac{v}{E}=\frac{z_{e f f}}{6 \pi \eta \cdot R_{h y d}}
$$

These variables can be controlled in the separation by altering the properties of the background electrolyte (BGE) depending on the intrinsic chemical structure of the analyte. $Z_{\mathrm{eff}}$ is determined through the charge on a weakly ionic analyte, which can be altered by changing buffer $\mathrm{pH}$ conditions of the BGE based on the $\mathrm{pK}_{\mathrm{a}}$ of the functional group moiety. The ionic strength of the BGE can modulate $\mathrm{z}_{\mathrm{eff}}$, where an increased ionic strength can decrease $\mu_{\mathrm{ep}}$ by counter-ions forming an electric double layer that reduce the effective charge of the ion. $\mathbf{R}_{\text {hyd }}$ is related to the hydrated molecular volume of a solvated ion. The ability of CE to separate different analytes is 
based primarily on differences in their effective charge density or $\mu_{\text {ep. }}$. Thus, the composition of the BGE plays a critical role in the selectivity of the separation process in CE.

The EOF is classified as the bulk flow of solution inside the capillary created by application of an external electric field. The surface of the capillary has silanol groups which are weakly acidic and undergo ionization in contact with an aqueous electrolyte solution buffers, resulting in a negatively charged surface under most $\mathrm{pH}$ conditions $(\mathrm{pH}>2)$. The positive ions of the electrolyte will thus form a rigid layer of ions adsorbed onto the capillary wall, while also forming a diffuse electric double layer from the capillary surface into bulk solution (Yan, 1996; Figure 1.3). When an electric field is applied across the capillary, the cations in the diffuse layer migrate towards the cathode, creating a natural electrokinetic pumping mechanism referred to as the EOF. Unlike chromatographic separations that require an external pump for solution flow, the EOF serves as an intrinsic flow that carries all species (neutral, cation, anion) towards to a detector. Since the EOF has a flat flow profile which decreases axial diffusion/band broadening, separation efficiency is enhanced in CE relative to parabolic hydrodynamic flow in HPLC. If the BGE is highly acidic $(\mathrm{pH}<2)$, then the EOF is highly suppressed because the silanol groups are not deprotenated and become predominately neutral without formation of an electric double layer. Similar to $\mu_{\mathrm{ep}}$, the magnitude of the EOF is primarily determined by the buffer $\mathrm{pH}$ and ionic strength of the BGE, where high $\mathrm{pH}$ and low ionic strength conditions favour a strong cathodic EOF.

These two main forces in the CE separation contribute to the overall mobility of the analyte in the separation. The apparent mobility $\left(\mu_{\text {ep }}^{\mathrm{A}}\right)$ of an analyte is the vector sum of $\mu_{\mathrm{ep}}$ and $\mu_{\mathrm{EOF}}$, such that: 


$$
\mu_{\text {ep }}^{\mathrm{A}}=\mu_{\mathrm{ep}}+\mu_{\mathrm{EOF}}
$$

Determining these parameters, particularly $\mu_{\mathrm{ep}}$, gives intrinsic characteristics of the analyte that improves method precision, as EOF can vary with natural changes to the capillary wall, as well as with random differences in BGE composition. In most cases, a neutral EOF marker is added to the sample as a way to determine $\mu_{\mathrm{EOF}}$ in order to calculate $\mu_{\mathrm{ep}}$ from a typical electropherogram, which measures changes in detector response (e.g., UV absorbance) as a function of migration time. In general, separation optimization in CE involves maximizing resolution of analyte peaks while reducing analysis times based on changes in the composition of the BGE, capillary dimensions and applied voltage. High external voltages (up to $30 \mathrm{kV}$ ) allows for fast analysis times with minimal band dispersion in CE due to its linear dependence on both $\mu_{\mathrm{ep}}$ and $\mu_{\mathrm{EOF}}$ provided that adequate heat dissipation is achieved within the capillary.

\subsubsection{Enzymatic kinetics by $\mathrm{CE}$}

The major benefit of $\mathrm{CE}$ is its adjustable selectivity that allows for resolution of various classes of charged analytes (e.g., metals, metabolites, protein/DNA, intact cells) in complex sample mixtures. Neutral analytes can also be separated by CE with the addition of charged micelles (e.g., sodium dodecyl sulphate) in the BGE based on their differential partitioning during electromigration (Terabe, 2009). Thus, the separation environment can be readily changed to modulate analyte migration behaviour via their direct ionization and/or specific biomolecular interaction with charged additives in the BGE, such as complexing agents and antibodies. (Yan, 1996). Because of this flexibility, CE has become increasingly popular in many bio-analytical fields, with uses ranging from enzyme kinetics, chiral separations to DNA sequencing (Anzenbacher and Hudecek, 2001; Dovichi and Zhang, 2000; Glatz, 2006; Ha et al., 2006; 
Portmann et al., 2010; Prost and Thormann, 2003; Ptolemy and Britz-McKibbin, 2008; Rabe et al., 2008; Suntornsuk).

In particular, separation used for monitoring substrate and/or product turn-over rates in enzymatic reactions with $\mathrm{CE}$ has become increasingly popular. This is relevant in cases when products generated are stereoisomers (e.g., diastereomers, enantiomers) with similar spectroscopic properties that excludes conventional absorbance or fluorescence methods (Gavina et al., 2010). Many studies have used CE to examine enzymatic activity with off-line stopped reactions or on-line using the capillary as a microreactor in electrophoretically mediated microanalysis (EMMA; Glatz, 2006; Zhang et al., 2008a). The basic principle behind EMMA is the ability of the CE instrument to inject distinct plugs of reactant into the capillary and mix them together with pressure or voltage for an in-capillary reaction. The reaction can then be stopped by addition of an on-line quenching step (short contact mode), or the reaction is allowed to occur during the entire separation if the BGE is compatible with the enzyme reaction conditions (long contact mode; Fan and Scriba, 2010). The ability to control mixing of nanolitre volumes of reagents directly in capillary for chemical labelling also increases the usefulness of the method (Ptolemy and Britz-McKibbin, 2006). The benefit of using CE for enzyme based analysis includes high selectivity, short analysis times, low operating costs, with on-line reactions greatly increasing the versatility and automation (Zhang et al., 2008a). Although there have been studies investigating CYP activity using CE (Bhoopathy et al., 2001; Ha et al., 2006; Portmann et al., 2010), only a few attempts of measuring CYP activity based on NADPH consumption via CE have been reported (Zhang et al., 2008b; Zhang et al., 2006). The results of these studies showed promise as a label-free method for assessing CYP activity by measuring 
apparent turnover rates of $\mathrm{NADPH}$ and $\mathrm{NADP}^{+}$for well-characterized substrate probes that do not induce significant uncoupling of the catalytic cycle.

\subsection{Research Goals and Questions}

The overarching goal of this project was to develop a method that is able to measure CYP activity for any substrate or isoform based on monitoring the turn-over rates of NADPH/NADP ${ }^{+}$ during enzyme incubation by CE with UV absorbance detection. Because all CYP reactions consume and form NADPH and $\mathrm{NADP}^{+}$, respectively, this method aims to identify potential CYP substrates by determining CYP reactivity for substrates, verified by NADPH consumption. This would narrow down the amount of CYPs and substrates needed to be screened for substrate and metabolite identification with LC/GC/CE-MS, where method development can be expensive and labour intensive for each substrates and metabolite of interest. This benefits those trying to identify novel CYP function by finding substrates for poorly characterized isoforms, as well as discovering interaction in CYP mediated drug screening where many novel substrates need to have clear CYP activity identified. Because the method aims to be substrate and CYP isoform independent, it provides a flexible yet powerful way to identify CYP activity.

The objective of this project was to use heterologously expressed CYP1s and established fluorometric methods to develop a label free method for measuring CYP1 activity using CE. Zebrafish CYP1 proteins have been previously expressed and their activity towards several typical CYP1 substrates and standard fluorescent based plate assays had been determined (Scornaienchi et al., 2010a; Scornaienchi et al., 2010b). CYP1 activity was determined based on $\mathrm{NADPH} / \mathrm{NADP}^{+}$in an offline reaction with fluorescent substrates, rates of metabolism from CE and established fluorometric plate assays were compared (Chapter 2). The rates of cofactor consumption and product formation were used to determine the coupling efficiency with regards 
to substrate structure and isoform activity. Since poor coupling efficiencies may be expected for CYPs involved in xenobiotic metabolism, as the flexibility of their active sites allow various substrates to dock and start the catalytic cycle, the second objective was to develop a CE assay to determine if excessive cofactor consumption is due to ROS production using the fluorescent probe dichlorofluorescein (DCF, Chapter 3). This assay was developed to separate the fluorescent product formed when DCF reacts with ROS, while maintaining clear separation of NADPH/NADP ${ }^{+}$. This would allow for a single assay to be used to determine CYP activity, but correcting for excessive cofactor consumption based on the extent of ROS production.

We were able to use NADPH and $\mathrm{NADP}^{+}$as quantitative parameters associated with CYP activity and uncoupling, and showed that $O$-dealkylation of 7 -ethoxyresorufin by CYP1A had product formation rates similar to NADP+ production (Chapter 2). However, the vast majority of CYP mediated reactions showed excessive cofactor consumption, which we hypothesised was due to uncoupling. Preliminary data using CE-UV for identification of ROS production was able to resolve various dichlorofluorescein (DCF) products, however, the sensitivity was inadequate for measuring response changes in a CYP mediated reaction (Chapter 3). ROS production from CYP reactions was verified using a fluorometric kinetic assay, where trends of excessive cofactor consumption were confirmed with DCF production in uncoupled reactions (Chapter 2). Overall, this research aims to gain a better understanding of cofactor consumption in CYP reactions, while acquiring a better understanding of CYP1 activity, especially with regards to CYP1D1 which has little known function. 


\section{References:}

Afri, M., Frimer, A. A. and Cohen, Y. (2004). Active oxygen chemistry within the liposomal bilayer. Part IV: Locating 2',7'-dichlorofluorescein (DCF), 2',7'-dichlorodihydrofluorescein (DCFH) and 2',7'-dichlorodihydrofluorescein diacetate (DCFH-DA) in the lipid bilayer. Chem Phys Lipids 131, 12333.

Ansede, J. H. and Thakker, D. R. (2004). High-throughput screening for stability and inhibitory activity of compounds toward cytochrome P450-mediated metabolism. J Pharm Sci 93, 239-55.

Anzenbacher, P. and Hudecek, J. (2001). Differences in flexibility of active sites of cytochromes $\mathrm{P} 450$ probed by resonance Raman and UV-Vis absorption spectroscopy. J Inorg Biochem 87, 209-13.

Bhoopathy, S., Sarkar, M. and Thomas Karnes, H. (2001). A direct injection capillary electrophoretic technique for miniaturized high-throughput metabolic screening of the CYP 3A4 enzyme using quinidine as a probe. J Pharm Biomed Anal 25, 721-9.

Chen, X., Zhong, Z., Xu, Z., Chen, L. and Wang, Y. (2010). 2',7'-Dichlorodihydrofluorescein as a fluorescent probe for reactive oxygen species measurement: Forty years of application and controversy. Free Radic Res 44, 587-604.

Degregorio, D., Sadeghi, S. J., Di Nardo, G., Gilardi, G. and Solinas, S. P. (2011). Understanding uncoupling in the multiredox centre P450 3A4-BMR model system. J Biol Inorg Chem 16, 109-16.

Denisov, I. G., Makris, T. M., Sligar, S. G. and Schlichting, I. (2005). Structure and chemistry of cytochrome P450. Chem Rev 105, 2253-77.

Dike, M., Guarino, R., Keith, S. and Timmins, M. (2000). Development of a high throughput, fluorescent assay system to determine relative rates of metabolism. Drug Metab Rev 32, 183

Diotel, N., Le Page, Y., Mouriec, K., Tong, S. K., Pellegrini, E., Vaillant, C., Anglade, I., Brion, F., Pakdel, F., Chung, B. C. et al. (2010) Aromatase in the brain of teleost fish: expression, regulation and putative functions. Front Neuroendocrinol 31, 172-92.

Dovichi, N. J. and Zhang, J. (2000). How Capillary Electrophoresis Sequenced the Human Genome. Angew Chem Int Ed Engl 39, 4463-4468.

Fan, Y. and Scriba, G. K. (2010). Advances in-capillary electrophoretic enzyme assays. $J$ Pharm Biomed Anal 53, 1076-90.

Gavina, J. M., White, C. E., Finan, T. M. and Britz-McKibbin, P. (2010). Determination of 4hydroxyproline-2-epimerase activity by capillary electrophoresis: A stereoselective platform for inhibitor screening of amino acid isomerases. Electrophoresis 31, 2831-7.

Glatz, Z. (2006). Determination of enzymatic activity by capillary electrophoresis. J Chromatogr B Analyt Technol Biomed Life Sci 841, 23-37.

Goldstone, J. V., Goldstone, H. M., Morrison, A. M., Tarrant, A., Kern, S. E., Woodin, B. R. and Stegeman, J. J. (2007). Cytochrome P450 1 genes in early deuterostomes (tunicates and sea urchins) and vertebrates (chicken and frog): origin and diversification of the CYP1 gene family. Mol Biol Evol 24, 2619-31.

Goldstone, J. V., Jonsson, M. E., Behrendt, L., Woodin, B. R., Jenny, M. J., Nelson, D. R. and Stegeman, J. J. (2009). Cytochrome P450 1D1: a novel CYP1A-related gene that is not transcriptionally activated by PCB126 or TCDD. Arch Biochem Biophys 482, 7-16. 
Gorsky, L. D., Koop, D. R. and Coon, M. J. (1984). On the stoichiometry of the oxidase and monooxygenase reactions catalyzed by liver microsomal cytochrome P-450. Products of oxygen reduction. J Biol Chem 259, 6812-7.

Groeger, G., Quiney, C. and Cotter, T. G. (2009). Hydrogen peroxide as a cell-survival signaling molecule. Antioxid Redox Signal 11, 2655-71.

Guengerich, F. P. (2001). Common and uncommon cytochrome P450 reactions related to metabolism and chemical toxicity. Chem Res Toxicol 14, 611-50.

Ha, P. T., Sluyts, I., Van Dyck, S., Zhang, J., Gilissen, R. A., Hoogmartens, J. and VanSchepdael, A. (2006). Chiral capillary electrophoretic analysis of verapamil metabolism by cytochrome P450 3A4. J Chromatogr A 1120, 94-101.

Hamdane, D., Xia, C., Im, S. C., Zhang, H., Kim, J. J. and Waskell, L. (2009). Structure and function of an NADPH-cytochrome P450 oxidoreductase in an open conformation capable of reducing cytochrome P450. J Biol Chem 284, 11374-84.

Huang, Q. and Szklarz, G. D. (2010). Significant increase in phenacetin oxidation on L382V substitution in human cytochrome P450 1A2. Drug Metab Dispos 38, 1039-45.

Jonsson, M. E., Berg, C., Goldstone, J. V. and Stegeman, J. J. (2011). New CYP1 genes in the frog Xenopus (Silurana) tropicalis: induction patterns and effects of AHR agonists during development. Toxicol Appl Pharmacol 250, 170-83.

Kawai, Y.K., Ikenaka, Y., Fujita, F., and Ishizuka, M. (2010). The CYP1D subfamily of genes in mammals and other vertebrates. Mamm. Genome 21, 320 - 329.

Lewis, D. F., Lake, B. G. and Dickins, M. (2004). Quantitative structure-activity relationships within a homologous series of 7-alkoxyresorufins exhibiting activity towards CYP1A and CYP2B enzymes: molecular modelling studies on key members of the resorufin series with CYP2C5-derived models of human CYP1A1, CYP1A2, CYP2B6 and CYP3A4. Xenobiotica 34, 501-13.

Martignoni, M., Groothuis, G. and de Kanter, R. (2006a). Comparison of mouse and rat cytochrome P450-mediated metabolism in liver and intestine. Drug Metab Dispos 34, 1047-54.

Martignoni, M., Groothuis, G. M. M. and de Kanter, R. (2006b). Species differences between mouse, rat, dog, monkey and human CYP-mediated drug metabolism, inhibition and induction. Expert Opinion on Drug Metabolism \& Toxicology 2, 875-894.

Nebert, D. W., Dalton, T. P., Okey, A. B. and Gonzalez, F. J. (2004). Role of aryl hydrocarbon receptor-mediated induction of the CYP1 enzymes in environmental toxicity and cancer. $J$ Biol Chem 279, 23847-50.

Nelson, D. R., Koymans, L., Kamataki, T., Stegeman, J. J., Feyereisen, R., Waxman, D. J., Waterman, M. R., Gotoh, O., Coon, M. J., Estabrook, R. W. et al. (1996). P450 superfamily: update on new sequences, gene mapping, accession numbers and nomenclature. Pharmacogenetics 6, 1-42.

Papapostolou, I., Patsoukis, N. and Georgiou, C. D. (2004). The fluorescence detection of superoxide radical using hydroethidine could be complicated by the presence of heme proteins. Anal Biochem 332, 290-8.

Portmann, S., Kwan, H. Y., Theurillat, R., Schmitz, A., Mevissen, M. and Thormann, W. (2010). Enantioselective capillary electrophoresis for identification and characterization of human cytochrome P450 enzymes which metabolize ketamine and norketamine in vitro. J Chromatogr A 1217, 7942-8. 
Prasad, J. C., Goldstone, J. V., Camacho, C. J., Vajda, S. and Stegeman, J. J. (2007). Ensemble modeling of substrate binding to cytochromes P450: analysis of catalytic differences between CYP1A orthologs. Biochemistry 46, 2640-54.

Prost, F. and Thormann, W. (2003). Capillary electrophoresis to assess drug metabolism induced in vitro using single CYP450 enzymes (Supersomes): application to the chiral metabolism of mephenytoin and methadone. Electrophoresis 24, 2577-87.

Ptolemy, A. S. and Britz-McKibbin, P. (2006). Sample preconcentration with chemical derivatization in capillary electrophoresis. Capillary as preconcentrator, microreactor and chiral selector for high-throughput metabolite screening. J Chromatogr A 1106, 7-18.

Ptolemy, A. S. and Britz-McKibbin, P. (2008). New advances in on-line sample preconcentration by capillary electrophoresis using dynamic pH junction. Analyst 133, 1643-8.

Rabe, K. S., Gandubert, V. J., Spengler, M., Erkelenz, M. and Niemeyer, C. M. (2008). Engineering and assaying of cytochrome P450 biocatalysts. Anal Bioanal Chem 392, 1059-73.

Ramadass, P., Meerarani, P., Toborek, M., Robertson, L. W. and Hennig, B. (2003). Dietary flavonoids modulate PCB-induced oxidative stress, CYP1A1 induction, and AhR-DNA binding activity in vascular endothelial cells. Toxicol Sci 76, 212-9.

Renwick, A. B., Surry, D., Price, R. J., Lake, B. G. and Evans, D. C. (2000). Metabolism of 7 benzyloxy-4-trifluoromethyl-coumarin by human hepatic cytochrome P450 isoforms. Xenobiotica 30, 955-69.

Schlezinger, J. J., Struntz, W. D., Goldstone, J. V. and Stegeman, J. J. (2006). Uncoupling of cytochrome P450 1A and stimulation of reactive oxygen species production by co-planar polychlorinated biphenyl congeners. Aquat Toxicol 77, 422-32.

Schlezinger, J. J., White, R. D. and Stegeman, J. J. (1999). Oxidative inactivation of cytochrome P-450 1A (CYP1A) stimulated by 3,3',4,4'-tetrachlorobiphenyl: production of reactive oxygen by vertebrate CYP1As. Mol Pharmacol 56, 588-97.

Scornaienchi, M. L., Thornton, C., Willett, K. L. and Wilson, J. Y. (2010a). Cytochrome P450-mediated 17beta-estradiol metabolism in zebrafish (Danio rerio). J Endocrinol 206, 317-25.

Scornaienchi, M. L., Thornton, C., Willett, K. L. and Wilson, J. Y. (2010b). Functional differences in the cytochrome P450 1 family enzymes from zebrafish (Danio rerio) using heterologously expressed proteins. Arch Biochem Biophys 502, 17-22.

Sligar, S. G. (1976). Coupling of spin, substrate, and redox equilibria in cytochrome P450. Biochemistry 15, 5399-406.

Smith, E. M. and Wilson, J. Y. (2010). Assessment of cytochrome P450 fluorometric substrates with rainbow trout and killifish exposed to dexamethasone, pregnenolone-16alpha-carbonitrile, rifampicin, and beta-naphthoflavone. Aquat Toxicol 97, 324-33.

Sue Masters, B. and Marohnic, C. C. (2006). Cytochromes P450--a family of proteins and scientists-understanding their relationships. Drug Metab Rev 38, 209-25.

Suntornsuk, L. Recent advances of capillary electrophoresis in pharmaceutical analysis. Anal Bioanal Chem 398, 29-52.

Terabe, S. (2009). Capillary separation: micellar electrokinetic chromatography. Annu Rev Anal Chem (Palo Alto Calif) 2, 99-120.

Uno, Y., Uehara, S., Murayama, N. and Yamazaki, H. (2011). CYP1D1, pseudogenized in human, is expressed and encodes a functional drug-metabolizing enzyme in cynomolgus monkey. Biochem Pharmacol 81, 442-50. 
Viswanathan, S., Hammock, B. D., Newman, J. W., Meerarani, P., Toborek, M. and Hennig, B. (2003). Involvement of CYP 2C9 in mediating the proinflammatory effects of linoleic acid in vascular endothelial cells. J Am Coll Nutr 22, 502-10.

Winterbourn, C. C. and Hampton, M. B. (2008). Thiol chemistry and specificity in redox signaling. Free Radic Biol Med 45, 549-61.

Xu, X. and Arriaga, E. A. (2009). Qualitative determination of superoxide release at both sides of the mitochondrial inner membrane by capillary electrophoretic analysis of the oxidation products of triphenylphosphonium hydroethidine. Free Radic Biol Med 46, 905-13.

Yan, X. (1996). Tutorial: Capillary Electrophoresis. The Chemical Educator 1, 14.

Zanette, J., Jenny, M. J., Goldstone, J. V., Woodin, B. R., Watka, L. A., Bainy, A. C. and Stegeman, J. J. (2009). New cytochrome P450 1B1, 1C2 and 1D1 genes in the killifish Fundulus heteroclitus: Basal expression and response of five killifish CYP1s to the AHR agonist PCB126. Aquat Toxicol 93, 234-43.

Zhang, J., Hoogmartens, J. and Van Schepdael, A. (2008a). Advances in CE-mediated microanalysis: an update. Electrophoresis 29, 56-65.

Zhang, J., Hoogmartens, J. and Van Schepdael, A. (2008b). Kinetic study of cytochrome P450 by capillary electrophoretically mediated microanalysis. Electrophoresis 29, 3694-700.

Zhang, J., Lou, Y., Hoogmartens, J. and Van Schepdael, A. (2006). Screening of drug metabolism by CE. Electrophoresis 27, 4827-35.

Zhao, H., Kalivendi, S., Zhang, H., Joseph, J., Nithipatikom, K., Vasquez-Vivar, J. and Kalyanaraman, B. (2003). Superoxide reacts with hydroethidine but forms a fluorescent product that is distinctly different from ethidium: potential implications in intracellular fluorescence detection of superoxide. Free Radic Biol Med 34, 1359-68. 

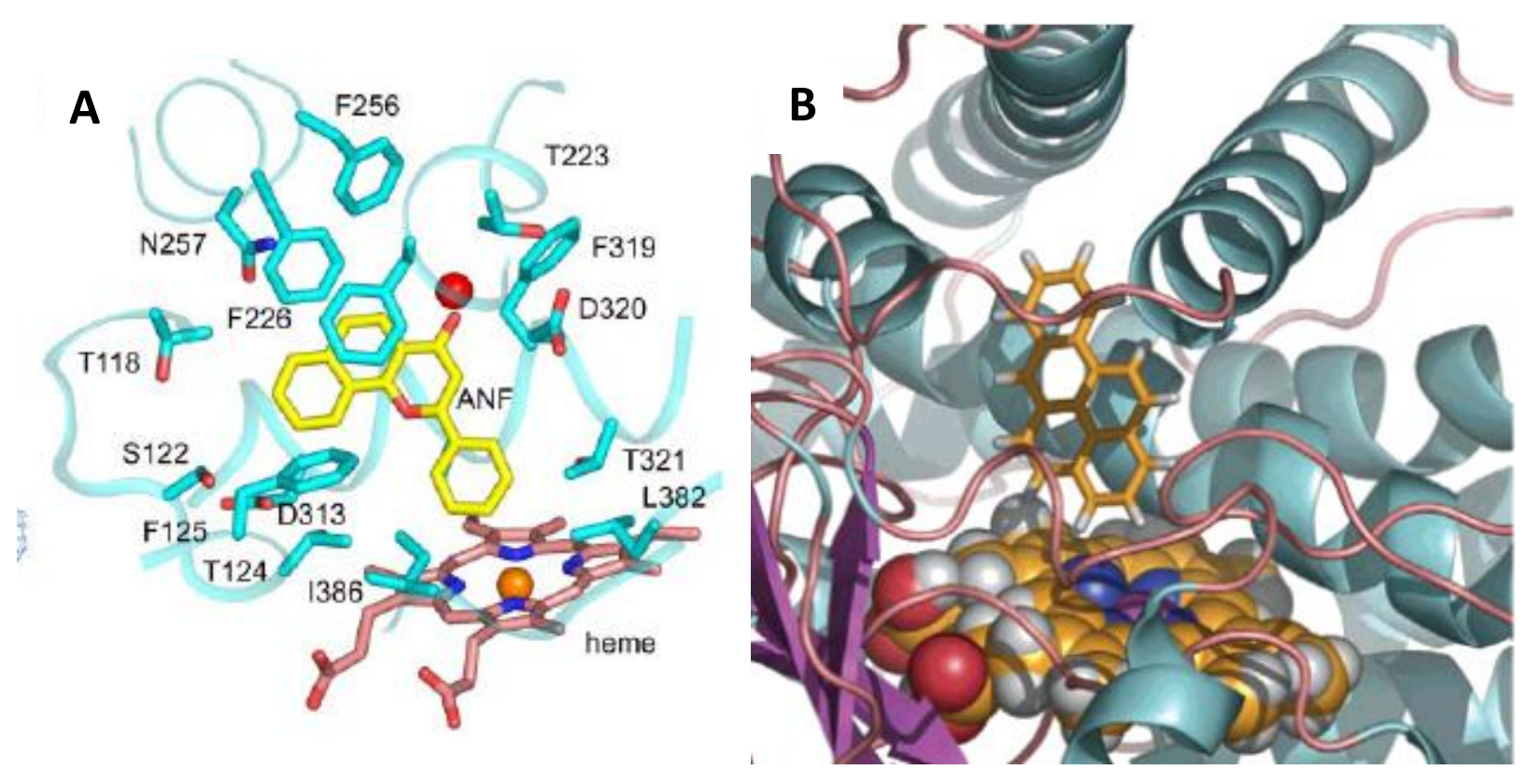

Figure 1.1: Binding of substrates near the heme of CYP enzymes. A) Binding of $\alpha$-naphthooflavone (ANF) into the active site of human CYP1A (Sansen et al, 2007), and B) probable B[a]P conformation into the fish CYP1A active site (Prasad et al., 2007). Displacement of a water molecule near the heme allows for metabolism if the compound in the active site is able to be transformed. 


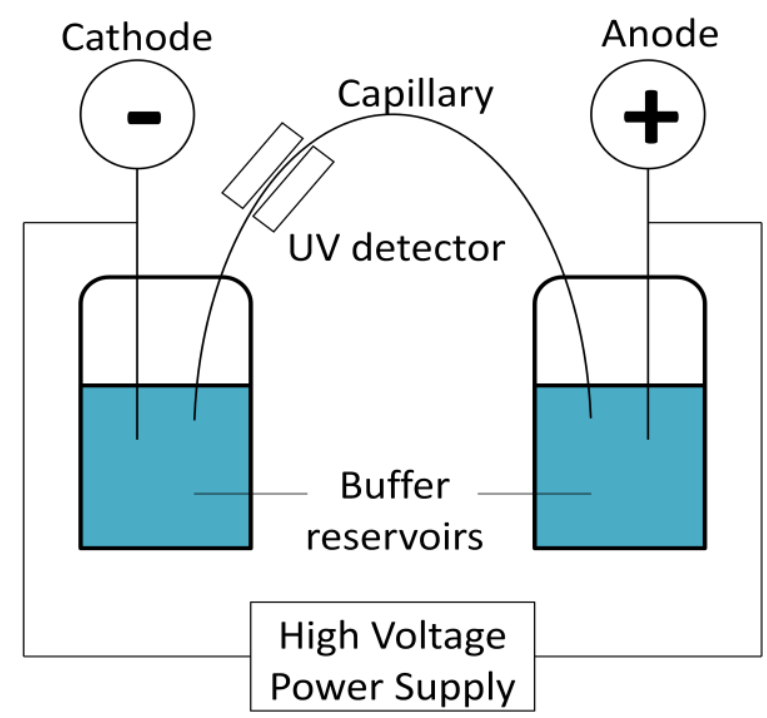

Figure 1.2 The general apparatus for capillary electrophoresis (CE). CE is comprised of a narrow fused-silica capillary, two aqueous buffer reservoirs, platinum electrodes connected to a high voltage supply and an on-line UV absorbance detector near the distal end of the capillary. 

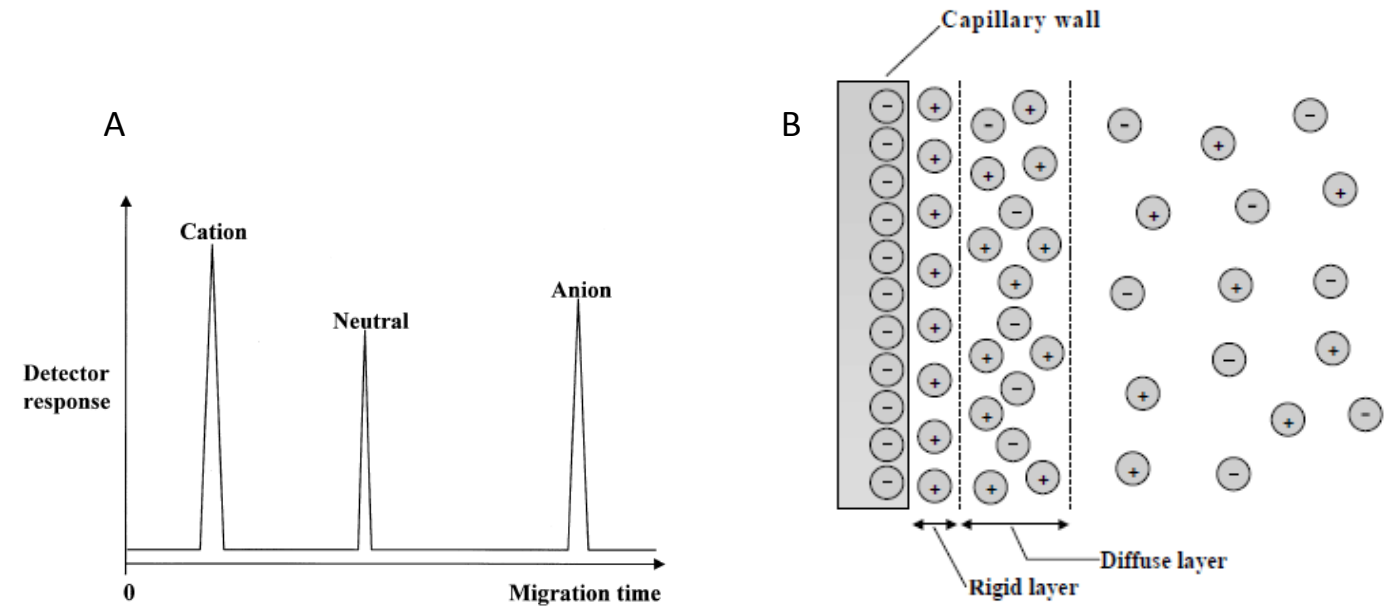

Figure 1.3: The migration order of ions and neutral compounds in a fused-silica capillary under

basic conditions. A) An electropherogram showing the order of ions migrating through the capillary under basic conditions, and B) The charge distribution inside the capillary with a basic BGE. The negative charge on the capillary wall allows for a rigid cation layer to form directly on the capillary wall. A diffuse layer of buffer ions beside the rigid layer allows for cations to migrate towards the cation, creating a bulk flow analogous to a pump in LC or GC which is the electroosmotic flow (EOF). Adapted from (Yan, 1996). 
MSc Thesis - J.G. Harskamp McMaster University - Biology 


\title{
Chapter 2:
}

\section{Functional Screening of Cytochrome P450 Activity and Uncoupling by Capillary Electrophoresis}

\author{
James Harskamp ${ }^{1}$, Philip Britz-McKibbin ${ }^{2, *}$ and Joanna Y. Wilson ${ }^{1, *}$ \\ ${ }^{1}$ Department of Biology and ${ }^{2}$ Department of Chemistry and Chemical Biology, McMaster \\ University, Hamilton, Ontario, Canada L8S 4M1
}

*Submitted to Analytical Chemistry “Letters”, this chapter has been modified in format from the submitted article

\section{* These authors have contributed equally; authors for correspondence:}

Dr. Joanna Y. Wilson, McMaster University, Department of Biology, Hamilton, ON, Canada L8S 4K1, 905-525-9104 (tel.), 905-522-6066 (fax), E-mail: joanna.wilson@ mcmaster.ca

Dr. Philip Britz-McKibbin, McMaster University, Department of Chemistry and Chemical Biology, McMaster University, Hamilton, ON, L8S 4M1, Canada, 905-525-9140 (tel.), E-mail: britz@mcmaster.ca

KEYWORDS Cytochrome P450 / Enzyme kinetics / Capillary electrophoresis / Alkoxyresorufin O-dealkylase / Uncoupling / CYP1 family/ Reactive oxygen species 


\begin{abstract}
Cytochrome P450s (CYPs) are functionally diverse monooxygenases responsible for oxidation of endogenous and xenobiotic compounds. The function of non-mammalian CYPs are largely unknown and tools for characterization limited. CYPs critical for xenobiotic metabolism are prone to catalytic cycle uncoupling resulting in reactive oxygen species (ROS) generation that is highly dependent on the specific CYP isoform and substrate interaction. This study describes the rapid assessment of the activity and coupling efficiency of CYPs using capillary electrophoresis with UV detection. The coupling efficiency of five zebrafish (Danio rerio) CYP1 isoforms with a series of fluorogenic substrate probes was determined by the rate of $\mathrm{NADP}^{+}$formation and compared with fluorescent product turn-over rates. In most cases, $\mathrm{NADP}^{+}$formation significantly overestimated CYP1 catalytic activity for substrate $O$-dealkylation suggesting uncoupling. ROS production was confirmed by elevated hydrogen peroxide generation in poorly coupled reactions. Reactions with $\beta$-estradiol confirmed that CYP1A, 1C1 and 1C2 have greater catalytic activity and coupling efficiency; CYP1B1 and 1D1 had coupling efficiencies under $4 \%$. This work highlights the wide disparity in uncoupling induced by unproductive substrate binding among CYP isoforms.
\end{abstract}




\subsection{Introduction}

Cytochrome P450 monooxygenases (CYPs) are heme-containing enzymes responsible for metabolism of endogenous compounds and xenobiotics (Denisov et al., 2005). Substrate binding triggers activation of molecular oxygen at the heme centre upon electron transfer from NADPH as mediated by flavoprotein reductases that result in regio- or stereoselective hydroxylation, dealkylation and epoxidation (Guengerich, 2007). CYPs can transform diverse substrates due to structural flexibility of their active site; human CYP3A4 has the most promiscuous active site and is responsible for the metabolism $\geq 50 \%$ of known pharmaceuticals (Ekroos and Sjogren, 2006). Although CYP-catalyzed reactions are associated with detoxification of non-polar substrates, reactive intermediates can induce oxidative stress and cell proliferation, such as $\beta$ estradiol 4-hydroxylation by CYP1B1 (Zhu and Lee, 2005). In several cases, CYPs have low coupling efficiencies in terms of electron flow along the redox chain resulting in loss of NADPH reducing equivalents and activated oxygen release without substrate modification (Degregorio et al., 2011; Gorsky et al., 1984). Catalytic uncoupling for CYPs can represent a major source of intra-cellular oxidative stress due to generation of reactive oxygen species (ROS; Green et al., 2008; Schlezinger et al., 2006). Thus, new methods that allow for rapid assessment of substrateinduced CYP uncoupling are needed to better understand its importance in enzyme inhibition, carcinogenesis and/or cytotoxicity.

High-throughput screening of CYP activity is performed using fluorogenic substrate probes, a cornerstone in drug development for in vitro assessment of therapeutic efficacy and drug interactions (Crespi and Stresser, 2000; Xu et al., 2010). These approaches are not effective for non-mammalian CYPs where functional characterizations are lacking and CYP substrate selectivity differs. Liquid chromatography-mass spectrometry (LC-MS) offers a more selective 
approach for detection of CYP-catalyzed oxidation product(s)(Youdim and Saunders, 2010) yet, optimization of separation and ionization conditions are required for each substrate and/or CYPderived product(s). The depletion of NADPH or production of $\mathrm{NADP}^{+}$may serve as a universal assay for CYP activity (Zhang et al., 2006). Due to background spectral interferences with heme protein, fluorescence monitoring of NADPH consumption during CYP-catalyzed reactions is not feasible without prior separation. Capillary electrophoresis (CE) offers a convenient microseparation platform for enzyme kinetic studies (Fan and Scriba, 2010) that benefits from low protein/reagent amounts, short analysis times, minimal sample pre-treatment and high selectivity notably for polar metabolites and their stereoisomers. (Portmann et al., 2010; Zhu and Lee, 2005).

For instance, the application of CE-based rates of $\mathrm{NADP}^{+}$generation to studies of CYPs without well characterized function will require an assessment of uncoupling because specific probes are lacking. CYP3A4 activity for testosterone and nifedipine measured by NADP ${ }^{+}$ generation with $\mathrm{CE}$, provided consistent results relative to conventional fluorescence inhibition without uncoupling (Zhang et al., 2008). However, compounds tested were well-characterized, specific CYP3A4 probes. Even in well characterized systems, other substrates and/or CYP isoforms can undergo significant uncoupling that can bias the stoichiometric relationship of $\mathrm{NADP}^{+}$to product turn-over rates due to release of superoxide anion $\left(\mathrm{O}_{2}{ }^{-}\right)$, hydrogen peroxide $\left(\mathrm{H}_{2} \mathrm{O}_{2}\right)$ and/or water $\left(\mathrm{H}_{2} \mathrm{O}\right)$ during the catalytic cycle (Manoj et al., 2010; Figure S1). Herein, we report the first systematic study of activity and coupling efficiency measured by $\mathrm{CE}$ among five zebrafish CYP1 isoforms (Scornaienchi et al., 2010a; Scornaienchi et al., 2010b) for a series of fluorogenic substrates (Table S1) based on comparison of the enzyme activity derived from rates 
of $\mathrm{NADP}^{+}$and product formation using $\mathrm{CE}$ and fluorescence, respectively. The zebrafish isoforms include those with well defined (e.g. CYP1A) and no defined (e.g. CYP1D1) function.

\subsection{EXPERIMENTAL}

\subsubsection{Chemicals and Reagents.}

Reagents and suppliers used for cloning and protein expression are described by Scornaienchi et al. (2010b). Reagents used for buffers were Tris-HCl, NaCl, Tris-acetate, ethylenediaminetetraacetic acid (EDTA), sucrose, and boric acid and were purchased from Fischer Scientific Inc. (Ottawa, Ontario). The resorufin fluorometric probes (7methoxyresorufin; 7MR, 7-ethoxyresorufin; 7ER, 7-pentoxyresorufin; 7PR, and 7benzyloxydresorufin; 7BR) were purchased from Sigma-Aldrich Inc. (Oakville, ON). 7benzyloxy-4-(trifluoromethyl)coumarin (BFC) and 3-cyano-7-ethoxycoumarin (CEC) were purchased from BD Gentest (Woburn, MA). NADPH, NADP ${ }^{+}$, 3,3,4,4-tetrachlorobiphenyl (PCB 77), $\beta$-estradiol ( $\left.\mathrm{E}_{2}\right)$, dichlorodihydrofluorescein diacetate (DCF-DA), dihydroethidium and $m$ nitrophenol $\left(\mathrm{m}-\mathrm{NO}_{2} \mathrm{Ph}\right)$ were purchased from Sigma.

\subsubsection{Preparation of Heterologously Expressed Recombinant Zebrafish CYP1s.}

Five modified zebrafish CYP1 genes (CYP1A, 1B1, 1C1, 1C2 and 1D1) were cloned into competent E. coli JM109 cells (Promega, Madison, WI) along with human cytochrome P450 reductase (CPR), and expressed proteins were harvested as described by Scornaienchi et al. (2010b). Membranes were tested for CYP activity, total P450 content, and total cytochrome c 
activity using alkoyxresorufin- $O$-dealkylation assays, a P450 carbon monoxide difference assay,

and a cytochrome-c reductase assay, respectively (Scornaienchi et al., 2010a). Membranes were stored at $-80^{\circ} \mathrm{C}$ and had small volumes chipped off the frozen preparation for fluorescence and CE experiments. Proteins used for enzyme kinetic studies were diluted into a membrane buffer (50 mM Tris-acetate, $0.25 \mathrm{mM}$ EDTA, $250 \mathrm{mM}$ sucrose, $\mathrm{pH}$ 7.6).

\subsubsection{CYP1 Activity Measurements Based on Product Turn-over Rates by Fluorescence.}

Six synthetic fluorescent substrates were chosen based on known CYP1 activity (7methoxyresorufin (MR), 7-ethoxyresorufin (ER), 7-pentoxyresorufin (PR), 7-benzyloxyresorufin (BR), 3-cyano-7-ethoxycoumarin (CEC) and 7-benzyloxy-4-(trifluoromethyl)coumarin (BFC) as shown in Table S1.(Scornaienchi et al., 2010b) The 7-ER used for kinetic assays was prepared using a saturated stock, using the extinction coefficient $22.5 \mathrm{M}^{-1} \mathrm{~cm}^{-1}$ at $482 \mathrm{~nm}$ to determine final solution concentration. The 7-MR, 7-PR, 7-BR, BFC, and CEC solutions were prepared from methanol or acetonitrile stock solutions and stored light protected at $-20{ }^{\circ} \mathrm{C}$. Substrates were prepared in assay buffer (50 mM Tris, $0.1 \mathrm{M} \mathrm{NaCl}, \mathrm{pH} 7.8)$, where initial substrate concentrations for were 3, 2, 3, 4, 10, $1000 \mu \mathrm{M}$ for 7-MR, 7-ER, 7-PR, 7-BR, CEC, and BFC respectively, optimized for multiwell plate fluorescence. CYP reactions with fluorescent substrates were initiated by adding freshly prepared (daily) NADPH to diluted CYP protein and substrate in buffer. The final [NADPH] was $20 \mu \mathrm{M}$. It should be noted that CYP and NADPH concentrations were 50 times lower than commonly used techniques, so that when measuring $\mathrm{NADPH} / \mathrm{NADP}^{+}$, excessive amounts of NADPH did not degrade due to auto-oxidation thereby increasing assay sensitivity (Zhang et al., 2006). Samples in duplicate had fluorescent product 
formation measured every 1.5 min by a fluorescence microplate reader (Synergy 2, Biotek Inc., VT, USA) directly after addition of NADPH at $28^{\circ} \mathrm{C}$ for $10 \mathrm{~min}$. Samples prepared for CE analysis followed the above procedure, however $12.5 \mu \mathrm{L}$ reaction aliquots were taken at $0,2.5,5$, 7.5, and 10 min for alkoxyresorufin substrates, and 0, 30, 60, 90, and 120 min for CEC, BFC and17ß-estradiol (E2) as rates were smaller for these latter substrates. These aliquots were then quenched in ice-cold methanol in a 1:1 ratio and kept on ice until CE analysis.

\subsubsection{CYP1 Activity Measurements Based on Rate of NADP+ Production by CE.}

Samples were kept on ice in the dark until moved to the sample tray which was kept at $4{ }^{\circ} \mathrm{C}$. All samples had $m$-nitrophenol as an internal standard added and were diluted to $100 \mu \mathrm{L}$ with a final Tris, $m$-nitrophenol and methanol concentration of $5 \mathrm{mM}, 35 \mu \mathrm{M}$ and $12.5 \% \mathrm{v} / \mathrm{v}$, respectively. Diluted methanol in samples used for enzyme quenching served as the neutral EOF marker. A 40 $\mathrm{cm}$ long fused-silica capillary (75 $\mu \mathrm{m}$ inner diameter, $11 \mathrm{~cm}$ effective length to detector) was used for separations at $25^{\circ} \mathrm{C}$ by $\mathrm{CE}$ (P/ACE MDQ, Beckman-Coulter Inc. Brea, CA, USA). The background electrolyte (BGE) was $100 \mathrm{mM}$ borate buffer, $\mathrm{pH}$ 9.2. Prior to each analysis, capillaries were flushed using a high pressure rinse (20 psi) for 1 min with $1 \mathrm{M} \mathrm{NaOH}$ followed by a 2 min flush with alkaline BGE. A $10 \mathrm{~s}$ hydrodynamic sample injection $(0.5 \mathrm{psi})$ loaded the sample into the short-end of the capillary outlet followed by application of voltage at $20 \mathrm{kV}$ under reverse polarity (outlet as cathode) together with an applied forward pressure (0.1 psi). This pressure-assisted reversed polarity separation format was developed to achieve short

analysis times with adequate resolution under 3 min when using CE with UV detection at 254 $\mathrm{nm}$ as depicted in Figure S2. Peak integration was performed using 32 Karat Software 
(Beckman Coulter, Inc.), where measured NADPH or $\mathrm{NADP}^{+}$responses were quantified relative to the internal standard. In all cases, the measured rates of $\mathrm{NADP}^{+}$formation used to assess CYP activity by $\mathrm{CE}$ was blank subtracted using a bacterial membrane containing only expressed CPR (no CYP1) to correct for background reductase activity that can both release and/or quench hydrogen peroxide with concomitant NADPH depletion (Manoj et al., 2010) as highlighted in

Figure S1. All enzyme kinetic studies were performed independently as inter-day biological replicates over 3 days with mean normalized $\mathrm{NADP}^{+}$responses measured as triplicate runs by CE.

\subsubsection{Fluorogenic Probes for ROS Detection.}

Detection of ROS for substrates $17 \beta$-estradiol $\left(\mathrm{E}_{2}\right)$ and 3,3,4,4-tetrachlorobiphenyl (PCB 77) was performed by using the fluorescent probes dihydroethidium $\left(\mathrm{H}_{2} \mathrm{E}\right)$ and dichlorofluorescein (DCF). ROS production was measured in E2 reactions with all CYP1 isoforms and reductase blanks (CPR); reactions with PCB 77 were only performed with CYP1A and CYP1D1 as a positive control. PCB 77 is a known uncoupler for fish CYP1A (Schlezinger et al., 2006) and CYP1D1 is the closest CYP1 homolog to CYP1A (Goldstone et al., 2009). A $200 \mu \mathrm{M}$ DCF working solution was prepared in $1.5 \mathrm{mM} \mathrm{NaOH}$ with a modified method described by Lebal et

al. (1992). HE reaction solution was $10 \mu \mathrm{M}$ HE made up from an ethanol stock. A $50 \mu \mathrm{M} \mathrm{E}_{2}$ with DCF (10 $\mu \mathrm{M}$ final reaction concentration) or $\mathrm{H}_{2} \mathrm{E}(10 \mu \mathrm{M}$ final reaction concentration) solution was made in reaction buffer ( $50 \mathrm{mM}$ tris, $0.1 \mathrm{M} \mathrm{NaCl}, \mathrm{pH} 7.8)$. Samples had CYP1 membranes diluted in membrane buffer (50 mM Tris-acetate, $0.25 \mathrm{mM}$ EDTA, $250 \mathrm{mM}$ sucrose, $\mathrm{pH}$ 7.6) added to the substrate, and the reactions were initiated with $1.4 \mathrm{mM} \mathrm{NADPH}$. Fluorescent 
product formation for DCF or HE was measured in a 96 well plate with an excitation/emission wavelength of $488 \mathrm{~nm} / 530 \mathrm{~nm}$ and $488 \mathrm{~nm} / 590 \mathrm{~nm}$, respectively.

\subsection{Results and Discussion}

Figure 1 depicts an overlay of electropherograms that highlights rapid assessment of $\mathrm{NADP}^{+}$and NADPH turn-over rates during CYP1A-catalyzed $O$-dealkylation of 7ethoxyresorufin (ER) when using a short-end injection together with pressure-assisted CE under reversed polarity with UV detection (Figure S2). Assay validation demonstrated short analysis times $(<3 \mathrm{~min})$, good reproducibility $(C V=3.2 \%)$, high linearity $\left(R^{2}>0.999\right)$ over a 100 -fold range with sub-micromolar detection limits $(S / N \approx 3,0.2 \mu \mathrm{M})$ for simultaneous quantification of $\mathrm{NADP}^{+}$and NADPH (Table S2 in Supplemental Information). An internal standard ( $m$ nitrophenol) added prior to $\mathrm{CE}$ analysis improved assay precision. Overall, $\mathrm{NADP}^{+}$derived data provided more reliable responses than NADPH due to its improved stability and was subsequently used for assessing CYP1 coupling efficiency (Figure S3).

A homologous series of $O$-alkoxyresorufin substrates with increasing side-chain length modulated CYP1A $O$-dealkylation as reflected by differences in the slopes of the NADP ${ }^{+}$linear response curves during enzyme incubation (i.e., ethyl $>$ pentyl $\approx$ methyl $>$ benzoyl: Figure 1 , inset). The high activity for 7-ethoxyresorufin (ER) by zebrafish CYP1A is consistent with its use as a selective probe for mammalian CYP1A1 with high binding affinity $\left(K_{m}=0.5 \mu \mathrm{M}\right.$; Lewis et al., 2007) and its widespread use as a marker of CYP1A activity and expression in fish. Recent studies on wild-type and mutant CYP1A1 variants have demonstrated the importance of aromatic $\pi-\pi$ interactions for ER docking in a catalytically favorable orientation for optimum product turnover (Lewis et al., 2007). In contrast, 7-methoxyresorufin (MR), 7-pentoxyresorufin (PR) and 7- 
benzoylresorufin (BR) are used as substrate probes for mammalian CYP1A2, CYP2B and/or CYP3A enzymes, respectively (Lewis et al., 2004). The two coumarin-based probes, 3-cyano7-ethoxycoumarin (CEC) and notably 7-benzyloxy-4-(trifluoromethyl)coumarin (BFC) with selectivity for mammalian CYP1A2, 3A4 and 2C9/19 (Renwick et al., 2000), had lower $O$ dealkylation activities by most zebrafish CYP1 isoforms thus requiring higher substrate concentrations and longer incubation times.

Zebrafish CYP1 activity based on product turn-over (fluorescence) relative to rates of $\mathrm{NADP}^{+}$generation $(\mathrm{CE})$ reflected a range of uncoupling among the fluorogenic substrates (Table 1). CYP coupling efficiency, estimated based on the mean rate of $\mathrm{NADP}^{+}$to product turn-over ratio (Traylor et al., 2011), ranged from 2-100\% (Figure 2). NADP $^{+}$generation often greatly overestimated actual product formation (Table 1) due to the significant CYP uncoupling in xenobiotic metabolism (Zangar et al., 2004). Overall, only four CYP isoform/substrate combinations had a coupling efficiency over $80 \%$; most reactions exhibited poor coupling $(<20 \%)$. CYP1A had $\sim 100 \%$ coupling efficiency for ER $O$-dealkylation and the highest product formation among all substrates. Zebrafish CYP1C2, a CYP1 isoform lacking in mammals, had $\sim 100 \%$ coupling efficiency for ER, but at a two-fold lower product turn-over rate relative to CYP1A. Unexpectedly, the closely related CYP1C1 had much lower coupling efficiency for ER than CYP1C2 and high coupling efficiency for CEC. In general, CYP1A showed broad and higher activity with good coupling for nearly all fluorogenic substrates with the exception of CEC and PR. The bulky yet conformational mobile long-chain alkoxyresorufin, PR displayed the lowest coupling efficiency for most CYP1 isoforms yet was the only substrate with appreciable catalytic activity for CYP1D1. CYP1D1, a pseudogene in humans, is functional in nonmammalian vertebrates (Goldstone et al., 2009; Zanette et al., 2009) and cynomolgus monkey 
(Uno et al., 2011). Zebrafish CYP1D1 had low activity for benzo[a]pyrene metabolism yet with distinct regioselectivity for hydroxylation unlike other CYP1 isoforms (Scornaienchi et al., 2010b). Cynomolgus monkey CYP1D1 had low activity for ER and caffeine 8-hydroxylation (Uno et al., 2011). MR, a mammalian CYP1A2 substrate, exhibited uncoupling for all CYP1s, including CYP1A; CYP1C2 had the highest coupling efficiency (43\%). The catalytic activity of all CYP isoforms for $O$-dealkylation of BR was low except for CYP1A, where high coupling efficiency was seen (86\%, Figure 2.) This work highlights the distinct selectivity, catalytic activity and coupling efficiency among zebrafish CYPs. To the best of our knowledge, this is the first study to have investigated the large disparity in coupling efficiency among various CYP isoforms for multiple substrates, which is difficult to model in silico due to the lack of available X-ray crystal structures and the wide statistical distributions of substrate binding configurations in the active site that impact metabolism (Prasad et al., 2007).

The catalytic activity and coupling efficiency of $17 \beta$-estradiol $\left(\mathrm{E}_{2}\right)$ hydroxylation, an endogenous substrate, with the CYP1 isoforms was examined using GC/MS data (Scornaienchi et al., 2010a) and CE. Previous studies have demonstrated that several hydroxylated regioisomers (i.e., 2-OH, 4-OH, $\alpha-16-\mathrm{OH}$ ) are generated by zebrafish CYP1s (Scornaienchi et al., 2010a); $\mathrm{E}_{2}$ hydroxylated regioisomers have widely different cellular activities in mammals including tumor inhibition and genotoxicity (Takemura et al., 2010; Zhu and Lee, 2005). Large variations in uncoupling were also found with $\mathrm{E}_{2}$ (Figure 3). Only CYP1A and CYP1C2 had high coupling efficiencies (89 and 97\%, respectively) although CYP1A had a 4-fold greater catalytic activity. CYP1D1 and CYP1B1 had the lowest hydroxylation rates with poor coupling (2.3 and 3.7\%, respectively). The excessive reducing equivalents of NADPH not utilized for product formation upon substrate binding was attributed largely to $\mathrm{H}_{2} \mathrm{O}_{2}$ release, based on 
dichlorofluorescein (DCF) as a $\mathrm{H}_{2} \mathrm{O}_{2}$-selective fluorogenic probe (Chen et al., 2010; Figure 3). Relative to CPR blanks (no CYP1), significantly higher peroxide responses were measured for poorly coupled enzyme reactions (i.e., CYP1D1 > 1C1 > 1B1) unlike CYP1A and CYP1C2. 1 $\mathrm{mM}$ ascorbic acid (AA) or ascorbic acid palmitate (AAP) as a hydrophilic and lipophilic antioxidant, respectively, attenuated $\mathrm{H}_{2} \mathrm{O}_{2}$ release during CYP1-catalyzed $\mathrm{E}_{2}$ hydroxylation, notably in the case of AA (Figure S4). Substrate-induced $\mathrm{H}_{2} \mathrm{O}_{2}$ generation via uncoupling of the catalytic cycle was confirmed using 3, 3', 4, 4'-tetrachlorobiphenyl (PCB77; Figure S5), which induces deleterious uncoupling of fish CYP1A (Schlezinger et al., 2006). Over a 230 and 13fold increase in the rate of peroxide generation was found with zebrafish CYP1A incubated with PCB77 $(10 \mu \mathrm{M})$ and $\mathrm{E}_{2}(50 \mu \mathrm{M})$, respectively relative to the CPR blank (no CYP1A). The primary ROS during PCB77 and $\mathrm{E}_{2}$-induced uncoupling was $\mathrm{H}_{2} \mathrm{O}_{2}$. No significant increase in $\mathrm{O}_{2}{ }^{-}$was found with dihydroethidium, a fluorogenic indicator of superoxide (Zhao et al., 2003; Figure S5). CPR has been reported to generate diffusable ROS while also functioning as an intrinsic $\mathrm{H}_{2} \mathrm{O}_{2}$ quencher in the absence of heme-containing CYP (Manoj et al., 2010), this effect was corrected in our studies by subtracting all responses with a CPR blank. Our work demonstrates that both endogenous compounds $\left(\mathrm{E}_{2}\right)$ and xenobiotics (PCB77) can promote oxidative stress via enhanced $\mathrm{H}_{2} \mathrm{O}_{2}$ production whose magnitude is dependent on the specific CYP isoform and the degree of electron transfer uncoupling. Indeed, amplification of ROS production may occur when persistent organic pollutants with low coupling efficiency induce CYP expression causing long-term oxidative stress that compromises intra-cellular antioxidant defence mechanisms (Gunawickrama et al., 2008). For instance, PCB77 binding of extra-hepatic CYP1B1 stimulated uncoupling with increased parameters of oxidative stress related to genotoxicity, including lipid peroxidation and DNA oxidation (Green et al., 2008). 
In summary, a systematic comparison of the catalytic activity and coupling efficiency of zebrafish CYP1 isoforms was performed with a series of non-fluorogenic and fluorogenic substrates. A selective yet rapid assay was developed for measuring $\mathrm{NADP}^{+}$or NADPH turnover rates in CYP-catalyzed reactions by CE and was used to determine the coupling efficiency for CYP1 isoforms. Substrate-induced uncoupling stimulated $\mathrm{H}_{2} \mathrm{O}_{2}$ release in most reactions, which was highly dependent on the substrate structure and CYP isoform. There is growing recognition that CYP uncoupling represents a major source of oxidative stress in the cell. Zebrafish CYP1A displayed broad selectivity, high activity and low uncoupling in contrast to CYP1D1, a CYP isoform with limited functional characterization (Scornaienchi et al., 2010a; Scornaienchi et al., 2010b; Uno et al., 2011). The measurement of $\mathrm{NADP}^{+}$production by CE provides a label-free approach to screen novel CYP isoforms for function although ROS production, specifically $\mathrm{H}_{2} \mathrm{O}_{2}$, must be measured concomitantly to prevent overestimation of catalytic activity. A fully quantitative model that corrects for all non-productive CYP uncoupling events was not feasible based on $\mathrm{NADP}^{+}$responses since a fraction of activated oxygen can lead to $\mathrm{H}_{2} \mathrm{O}$ release (undetected by ROS probes) that consumes two extra equivalents of NADPH relative to $\mathrm{O}_{2}{ }^{-}$or $\mathrm{H}_{2} \mathrm{O}_{2}$ without substrate modification (Gorsky et al., 1984). Future studies will develop an integrated CE method that can simultaneously monitor both cofactor and ROS during CYP-catalyzed reactions. Measurement of oxygen consumption in CYP-catalyzed reactions using selective electrodes (Huang and Szklarz, 2010) would allow for accurate assessment of catalytic activity and coupling efficiency in conjunction with CE. The application of multiplexed capillary arrays together with electrophoretically mediated microanalysis (Fan and Scriba, 2010) offers an automated yet high-throughput screening platform for functional screening of CYPs while consuming low amounts (pmol) of recombinant enzymes and chemical reagents. 


\section{Acknowledgements}

Financial support was from National Science and Engineering Research Council of Canada (NSERC) to P.B.M. and J.Y.W. J.H. received support in the form of the Canada Graduate Scholarship from NSERC. 


\section{References:}

Chen, X., Zhong, Z., Xu, Z., Chen, L. and Wang, Y. (2010). 2',7'-Dichlorodihydrofluorescein as a fluorescent probe for reactive oxygen species measurement: Forty years of application and controversy. Free Radic Res 44, 587-604.

Crespi, C. L. and Stresser, D. M. (2000). Fluorometric screening for metabolism-based drug-drug interactions. J Pharmacol Toxicol Methods 44, 325-31.

Degregorio, D., Sadeghi, S. J., Di Nardo, G., Gilardi, G. and Solinas, S. P. (2011). Understanding uncoupling in the multiredox centre P450 3A4-BMR model system. J Biol Inorg Chem 16, 109-16.

Denisov, I. G., Makris, T. M., Sligar, S. G. and Schlichting, I. (2005). Structure and chemistry of cytochrome P450. Chem Rev 105, 2253-77.

Ekroos, M. and Sjogren, T. (2006). Structural basis for ligand promiscuity in cytochrome P450 3A4. Proc Natl Acad Sci U S A 103, 13682-7.

Fan, Y. and Scriba, G. K. (2010). Advances in-capillary electrophoretic enzyme assays. J Pharm Biomed Anal 53, 1076-90.

Goldstone, J. V., Jonsson, M. E., Behrendt, L., Woodin, B. R., Jenny, M. J., Nelson, D. R. and Stegeman, J. J. (2009). Cytochrome P450 1D1: a novel CYP1A-related gene that is not transcriptionally activated by PCB126 or TCDD. Arch Biochem Biophys 482, 7-16.

Gorsky, L. D., Koop, D. R. and Coon, M. J. (1984). On the stoichiometry of the oxidase and monooxygenase reactions catalyzed by liver microsomal cytochrome P-450. Products of oxygen reduction. J Biol Chem 259, 6812-7.

Green, R. M., Hodges, N. J., Chipman, J. K., O'Donovan, M. R. and Graham, M. (2008). Reactive oxygen species from the uncoupling of human cytochrome P450 1B1 may contribute to the carcinogenicity of dioxin-like polychlorinated biphenyls. Mutagenesis 23, 457-63.

Guengerich, F. P. (2007). Mechanisms of cytochrome P450 substrate oxidation: MiniReview. J Biochem Mol Toxicol 21, 163-8.

Gunawickrama, S. H., Aarsaether, N., Orbea, A., Cajaraville, M. P. and Goksoyr, A. (2008). PCB77 (3,3',4,4'-tetrachlorobiphenyl) co-exposure prolongs CYP1A induction, and sustains oxidative stress in B(a)P-exposed turbot, Scophthalmus maximus, in a long-term study. Aquat Toxicol 89, 65-74.

Huang, Q. and Szklarz, G. D. (2010). Significant increase in phenacetin oxidation on L382V substitution in human cytochrome P450 1A2. Drug Metab Dispos 38, 1039-45.

LeBel, C. P., Ischiropoulos, H. and Bondy, S. C. (1992). Evaluation of the probe 2',7'dichlorofluorescin as an indicator of reactive oxygen species formation and oxidative stress. Chem Res Toxicol 5, 227-31.

Lewis, B. C., Mackenzie, P. I. and Miners, J. O. (2007). Comparative homology modeling of human cytochrome P4501A1 (CYP1A1) and confirmation of residues involved in 7-ethoxyresorufin Odeethylation by site-directed mutagenesis and enzyme kinetic analysis. Arch Biochem Biophys 468, 5869.

Lewis, D. F., Lake, B. G. and Dickins, M. (2004). Quantitative structure-activity relationships within a homologous series of 7-alkoxyresorufins exhibiting activity towards CYP1A and CYP2B enzymes: molecular modelling studies on key members of the resorufin series with CYP2C5-derived models of human CYP1A1, CYP1A2, CYP2B6 and CYP3A4. Xenobiotica 34, 501-13.

Manoj, K. M., Gade, S. K. and Mathew, L. (2010). Cytochrome P450 reductase: a harbinger of diffusible reduced oxygen species. PLoS One 5, e13272.

Portmann, S., Kwan, H. Y., Theurillat, R., Schmitz, A., Mevissen, M. and Thormann, W. (2010). Enantioselective capillary electrophoresis for identification and characterization of human cytochrome P450 enzymes which metabolize ketamine and norketamine in vitro. J Chromatogr $A$ 1217, 7942-8. 
Prasad, J. C., Goldstone, J. V., Camacho, C. J., Vajda, S. and Stegeman, J. J. (2007). Ensemble modeling of substrate binding to cytochromes P450: analysis of catalytic differences between CYP1A orthologs. Biochemistry 46, 2640-54.

Renwick, A. B., Surry, D., Price, R. J., Lake, B. G. and Evans, D. C. (2000). Metabolism of 7benzyloxy-4-trifluoromethyl-coumarin by human hepatic cytochrome P450 isoforms. Xenobiotica 30, 955-69.

Schlezinger, J. J., Struntz, W. D., Goldstone, J. V. and Stegeman, J. J. (2006). Uncoupling of cytochrome $\mathrm{P} 4501 \mathrm{~A}$ and stimulation of reactive oxygen species production by co-planar polychlorinated biphenyl congeners. Aquat Toxicol 77, 422-32.

Scornaienchi, M. L., Thornton, C., Willett, K. L. and Wilson, J. Y. (2010a). Cytochrome P450mediated 17beta-estradiol metabolism in zebrafish (Danio rerio). J Endocrinol 206, 317-25.

Scornaienchi, M. L., Thornton, C., Willett, K. L. and Wilson, J. Y. (2010b). Functional differences in the cytochrome P450 1 family enzymes from zebrafish (Danio rerio) using heterologously expressed proteins. Arch Biochem Biophys 502, 17-22.

Takemura, H., Itoh, T., Yamamoto, K., Sakakibara, H. and Shimoi, K. (2010). Selective inhibition of methoxyflavonoids on human CYP1B1 activity. Bioorg Med Chem 18, 6310-5.

Traylor, M. J., Chai, J. and Clark, D. S. (2011). Simultaneous measurement of CYP1A2 activity, regioselectivity, and coupling: Implications for environmental sensitivity of enzyme-substrate binding. Arch Biochem Biophys 505, 186-93.

Uno, Y., Uehara, S., Murayama, N. and Yamazaki, H. (2011). CYP1D1, pseudogenized in human, is expressed and encodes a functional drug-metabolizing enzyme in cynomolgus monkey. Biochem Pharmacol 81, 442-50.

Xu, R., Manuel, M., Cramlett, J. and Kassel, D. B. (2010). A high throughput metabolic stability screening workflow with automated assessment of data quality in pharmaceutical industry. J Chromatogr A 1217, 1616-25.

Youdim, K. A. and Saunders, K. C. (2010). A review of LC-MS techniques and high-throughput approaches used to investigate drug metabolism by cytochrome P450s. J Chromatogr B Analyt Technol Biomed Life Sci 878, 1326-36.

Zanette, J., Jenny, M. J., Goldstone, J. V., Woodin, B. R., Watka, L. A., Bainy, A. C. and Stegeman, J. J. (2009). New cytochrome P450 1B1, 1C2 and 1D1 genes in the killifish Fundulus heteroclitus: Basal expression and response of five killifish CYP1s to the AHR agonist PCB126. Aquat Toxicol 93, 234-43.

Zangar, R. C., Davydov, D. R. and Verma, S. (2004). Mechanisms that regulate production of reactive oxygen species by cytochrome P450. Toxicol Appl Pharmacol 199, 316-31.

Zhang, J., Hoogmartens, J. and Van Schepdael, A. (2008). Kinetic study of cytochrome P450 by capillary electrophoretically mediated microanalysis. Electrophoresis 29, 3694-700.

Zhang, J., Lou, Y., Hoogmartens, J. and Van Schepdael, A. (2006). Screening of drug metabolism by CE. Electrophoresis 27, 4827-35.

Zhao, H., Kalivendi, S., Zhang, H., Joseph, J., Nithipatikom, K., Vasquez-Vivar, J. and Kalyanaraman, B. (2003). Superoxide reacts with hydroethidine but forms a fluorescent product that is distinctly different from ethidium: potential implications in intracellular fluorescence detection of superoxide. Free Radic Biol Med 34, 1359-68.

Zhu, B. T. and Lee, A. J. (2005). NADPH-dependent metabolism of 17beta-estradiol and estrone to polar and nonpolar metabolites by human tissues and cytochrome P450 isoforms. Steroids 70, 22544. 


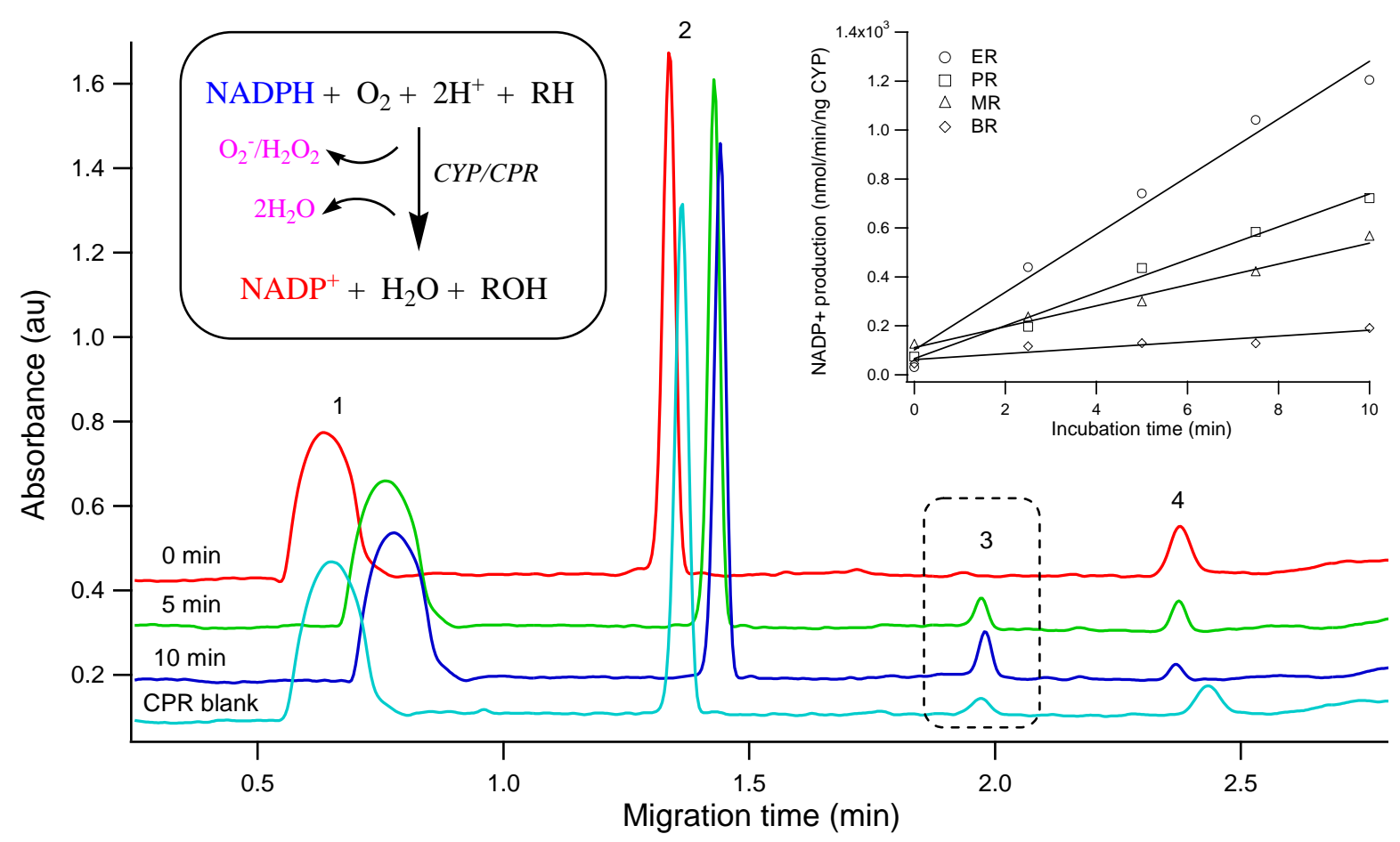

Figure 1. Assessment of zebrafish CYP1A activity for O-dealkylation of a homologous series of resorufin substrates (inset) using CE based on the rate of $\mathrm{NADP}^{+}$production relative to reductase blank (CPR at $10 \mathrm{~min}$ ). All CE runs were performed using a pressureassisted separation under reversed polarity with a short-end injection using $100 \mathrm{mM}$ borate, $\mathrm{pH}$ 9.2 with UV detection at $254 \mathrm{~nm}$. Enzyme kinetic studies were performed off-line at $28^{\circ} \mathrm{C}$ using recombinant CYP isoforms $(\sim 50 \mathrm{nM})$ in $50 \mathrm{mM}$ Tris, $100 \mathrm{mM} \mathrm{NaCl}, \mathrm{pH} 7.8$, which was initiated by NADPH $(20 \mathrm{mM})$ and quenched in ice-cold methanol. Peak numbering on electropherograms correspond to 1: EOF (methanol), 2: $m$-nitrophenol (internal standard), 3: $\mathrm{NADP}^{+}$and 4: NADPH. Linear increase in $\mathrm{NADP}^{+}$responses $\left(R^{2}>0.900\right)$ with enzyme incubation time reflects both coupled (productive) and uncoupled (non-productive) reaction pathways that is dependent both on substrate structure and specific CYP1 isoform. 
Table 1. Comparison of the catalytic activity of zebrafish CYP1s1 for six different fluorogenic substrates as measured by fluorescence and CE, respectively.

\begin{tabular}{|c|c|c|c|c|c|c|c|c|c|c|c|}
\hline \multirow[t]{2}{*}{ Substrate $^{2}$} & \multirow[t]{2}{*}{ Conc. $^{3}(\mu \mathrm{M})$} & \multicolumn{2}{|c|}{ CYP1A } & \multicolumn{2}{|c|}{ CYP1B1 } & \multicolumn{2}{|c|}{ CYP1C1 } & \multicolumn{2}{|c|}{ CYP1C2 } & \multicolumn{2}{|c|}{ CYP1D1 } \\
\hline & & Product & $N A D P^{+}$ & Product & $N A D P^{+}$ & Product & $N A D P^{+}$ & Product & $N A D P^{+}$ & Product & $N A D P^{+}$ \\
\hline MR & 3 & $10 \pm 1$ & $34 \pm 4$ & $0.7 \pm 0.1$ & $25 \pm 5$ & $1.4 \pm 1.1$ & $3.4 \pm 1.1$ & $2.3 \pm 0.8$ & $5 \pm 3$ & $0.1 \pm 0.1$ & $48 \pm 17$ \\
\hline ER & 2 & $61 \pm 22$ & $61 \pm 11$ & $12 \pm 1$ & $94 \pm 17$ & $1.0 \pm 0.3$ & $5.3 \pm 2.7$ & $29 \pm 15$ & $29 \pm 13$ & $n d$ & $11 \pm 6$ \\
\hline PR & 3 & $1.1 \pm 0.1$ & $46 \pm 6$ & $0.2 \pm 0.1$ & $68 \pm 26$ & $4.1 \pm 0.6$ & $n d$ & $0.3 \pm 0.1$ & $1.6 \pm 5.9$ & $1.3 \pm 0.1$ & $46 \pm 8$ \\
\hline $\mathrm{BR}$ & 4 & $7.7 \pm 0.5$ & $9 \pm 2$ & $1.8 \pm 0.1$ & $13 \pm 2$ & $0.3 \pm 0.1$ & $35 \pm 12$ & $n d$ & $n d$ & $0.1 \pm 0.05$ & $17 \pm 4$ \\
\hline CEC & 10 & $0.07 \pm 0.04$ & $0.5 \pm 0.2$ & $n d$ & $n d$ & $4.9 \pm 0.1$ & $4.9 \pm 0.1$ & $0.46 \pm 0.13$ & $2.00 \pm 0.12$ & $n d$ & $n d$ \\
\hline $\mathrm{BFC}$ & 1000 & $0.74 \pm 0.03$ & $1.6 \pm 0.2$ & $0.2 \pm 0.1$ & $1.6 \pm 0.1$ & $0.2 \pm 0.01$ & $1.90 \pm 0.03$ & $0.49 \pm 0.01$ & $2.97 \pm 0.05$ & $0.16 \pm 0.01$ & $1.90 \pm 0.06$ \\
\hline
\end{tabular}




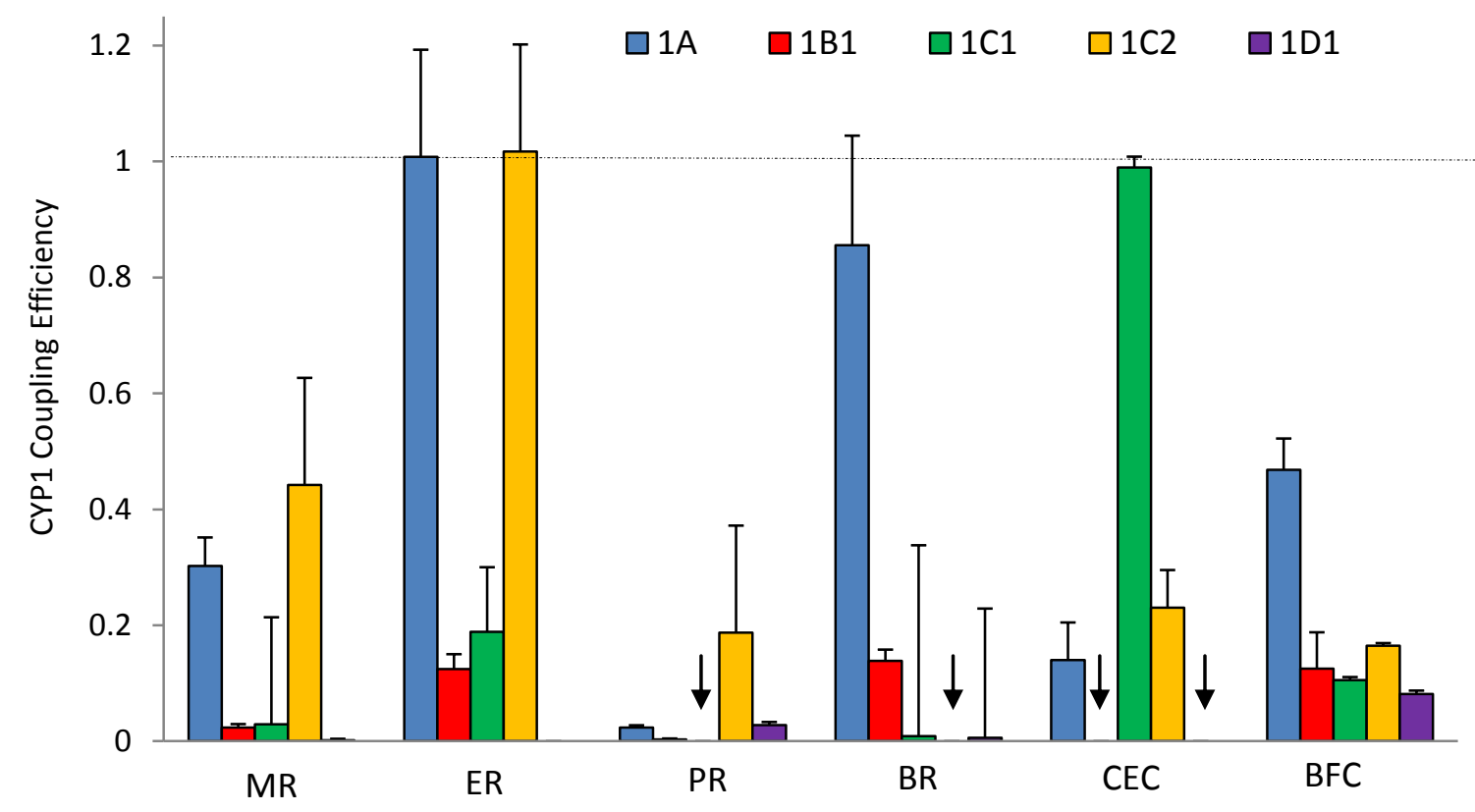

Figure 2. Substrate-dependence on apparent coupling efficiency $(\approx 0.3-100 \%)$ for recombinant CYP1 isoforms. Coupling efficiency is a ratio of measured rates of $\mathrm{NADP}^{+}$ generation (CE) relative to product formation (fluorescence) as summarized in Table 1. In general, electron transfer for CYP1A was efficiently coupled for $O$-dealkylation of most resorufin/coumarin substrates (except PR), whereas CYP1D1 overall had the lowest catalytic activity and coupling efficiency $(<3 \%)$. CYP1 isoforms with non-detectable activity for substrate conversion and/or $\mathrm{NADP}^{+}$generation under these conditions are denoted by arrows. 


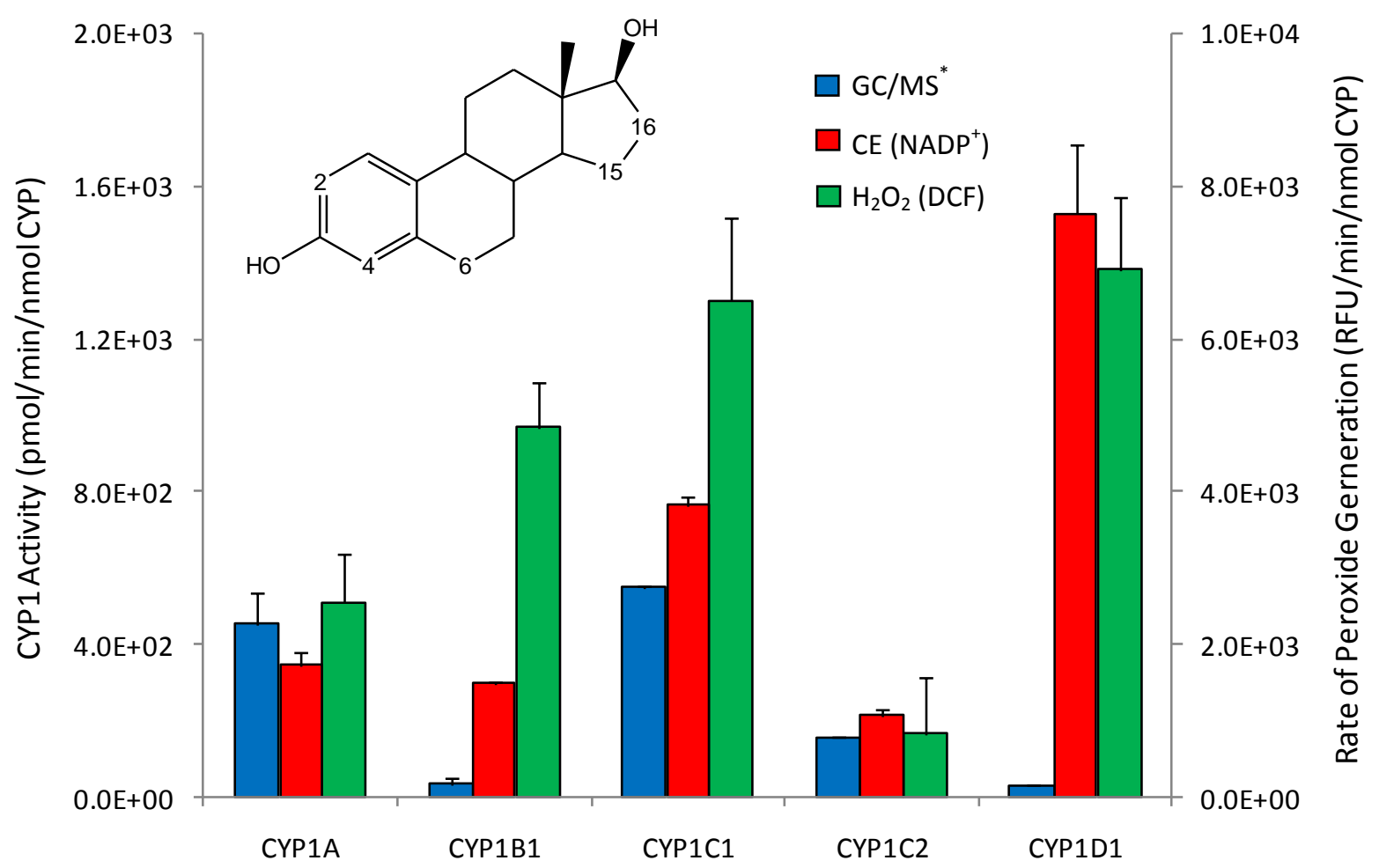

Figure 3. Activity of zebrafish CYP1 isoforms for 17ß-estradiol (50 mM) as a nonfluorogenic substrate when measuring rates of product formation by GC/MS, ${ }^{19} \mathrm{NADP}^{+}$ production by $\mathrm{CE}$ and $\mathrm{H}_{2} \mathrm{O}_{2}$ generation using dichlorofluorecein (DCF). Coupling efficiencies ranged from 2-96\%. In general, high coupling efficiencies were associated with lower levels of $\mathrm{H}_{2} \mathrm{O}_{2}$ (e.g., CYP1A, 1C2), whereas uncoupling resulted in greater rates of $\mathrm{H}_{2} \mathrm{O}_{2}$ production (e.g., CYP1C1, 1B1, 1D1). Regioselective oxidation of $17 \beta$-estradiol is denoted by numbers on the structure with hydroxylation at 2- and 4- positions being most common for zebrafish CYP1s. 


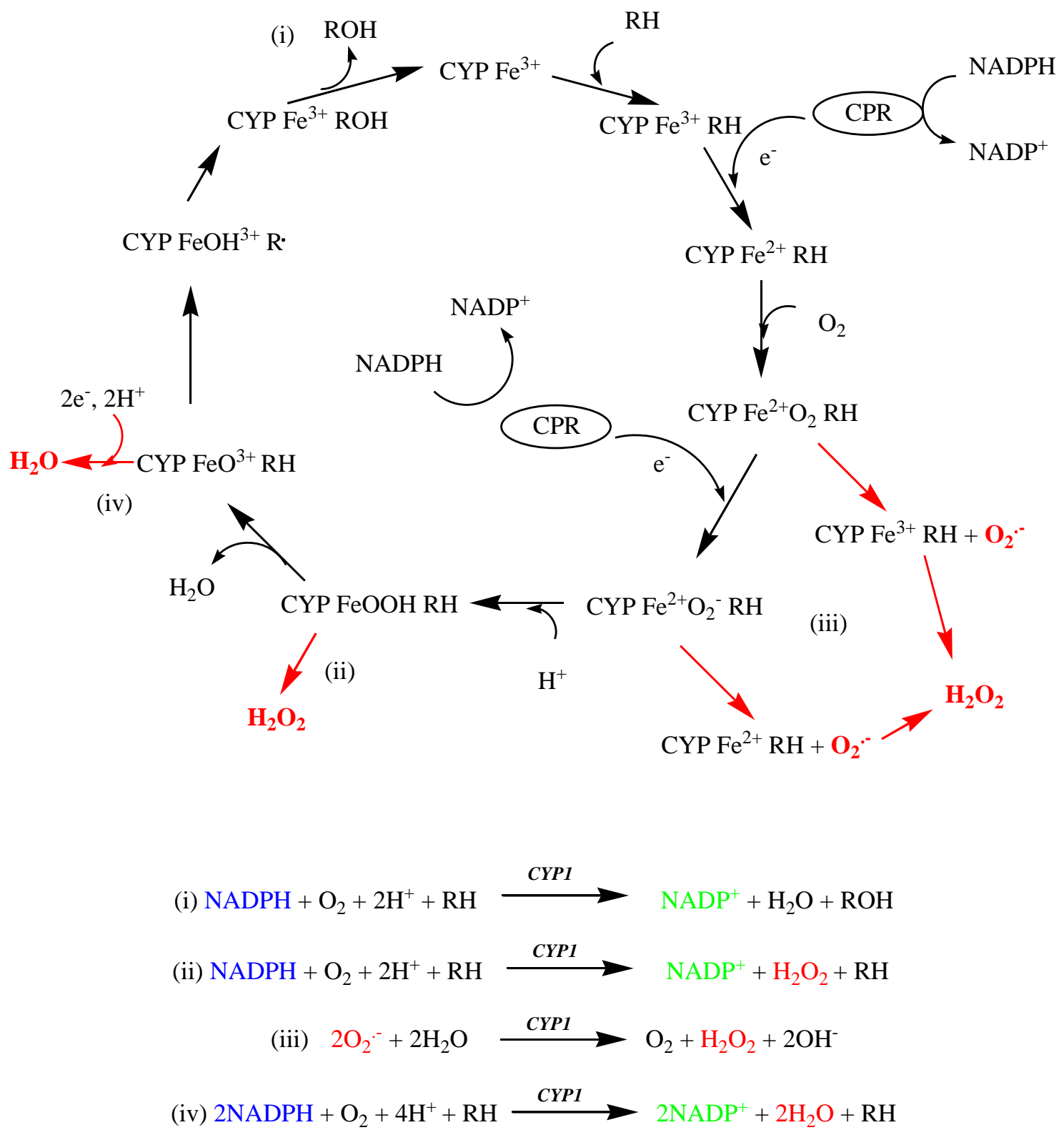

Figure S1. Multi-step redox cycle associated with enzyme-catalyzed oxidation of substrates by CYPs highlighting both coupled and uncoupled pathways that result in product formation or peroxide production, respectively. Equations describing NADPH consumption and ROS formation are linked to the catalytic cycle via the roman numerals assigned to them as modified from Guengerich. ${ }^{8}$ 
MSc Thesis - J.G. Harskamp McMaster University - Biology

Table S1. Structures and properties of synthetic fluorogenic probes used for assessment of CYP1 activity and coupling efficiency

\begin{tabular}{|c|c|c|c|c|c|}
\hline Substrate & Chemical structure & Product & $\begin{array}{c}\text { Excitation }^{a} \\
(\mathrm{~nm})\end{array}$ & $\begin{array}{c}\text { Emission }^{a} \\
(\mathrm{~nm})\end{array}$ & $\begin{array}{c}\text { Mammalian CYP } \\
\text { Specificity }^{b}\end{array}$ \\
\hline MR & & Resorufin & $530 / 20$ & $590 / 20$ & $1 \mathrm{~A} 2$ \\
\hline ER & & Resorufin & $530 / 20$ & $590 / 20$ & $1 \mathrm{~A} 1>1 \mathrm{~A} 2$ \\
\hline PR & & Resorufin & $530 / 20$ & $590 / 20$ & $2 \mathrm{~B} 6$ \\
\hline BR & & Resorufin & $530 / 20$ & $590 / 20$ & $2 \mathrm{~B}, 3 \mathrm{~A} 4$ \\
\hline $\mathrm{CEC}$ & & 3-Cyano-7-hydroxycoumarin & $400 / 30$ & $460 / 40$ & $1 \mathrm{~A} 1>1 \mathrm{~A} 2 \mathrm{C} 6,2 \mathrm{C} 11$ \\
\hline $\mathrm{BFC}$ & & $\begin{array}{l}\text { 7-Hydroxy-4-(trifluoromethyl) } \\
\text { coumarin }\end{array}$ & $400 / 30$ & $530 / 20$ & $3 \mathrm{~A} 4,1 \mathrm{~A} 2,2 \mathrm{C} 19$ \\
\hline
\end{tabular}




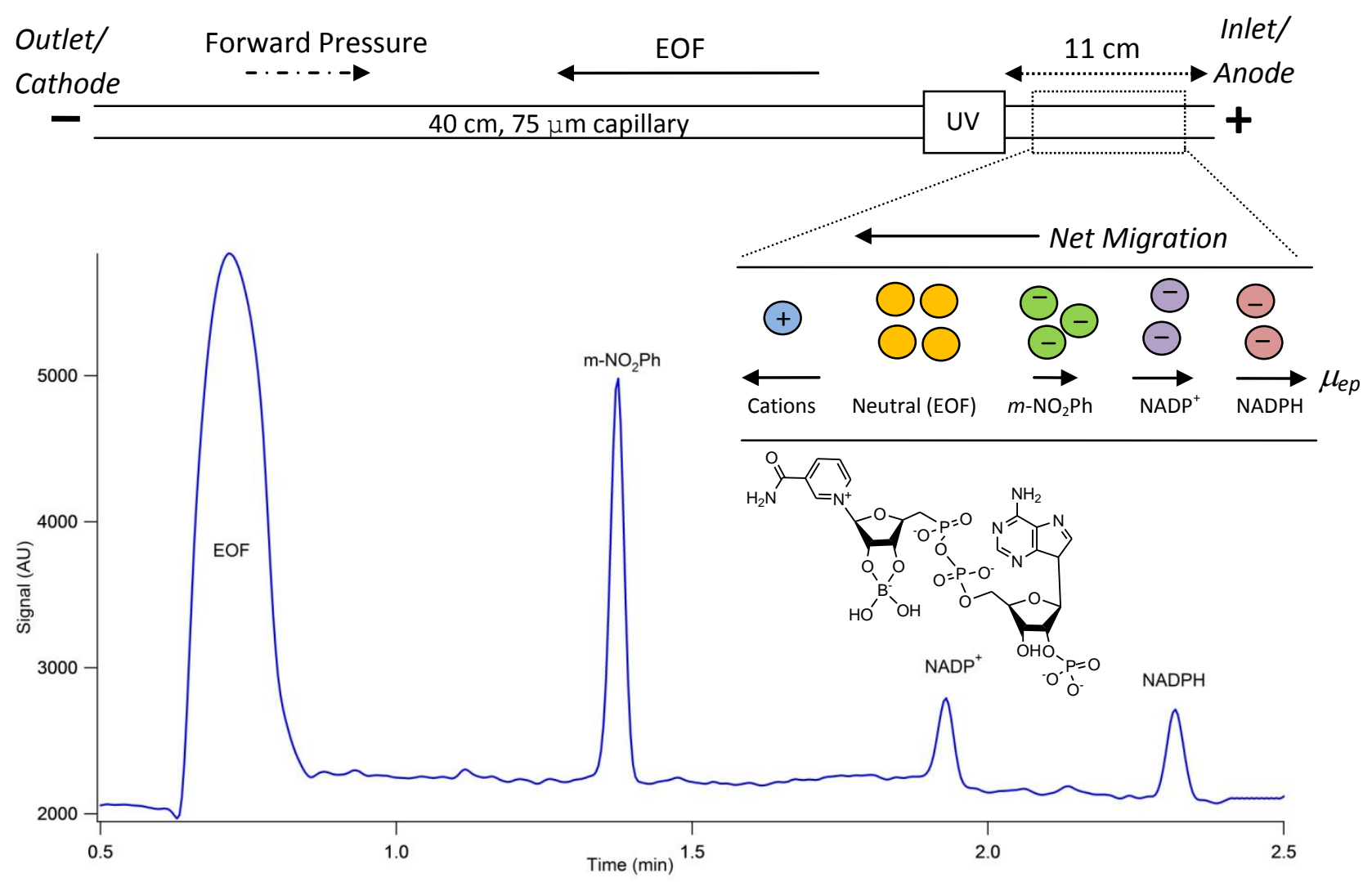

Figure S2. Scheme of rapid separation of cofactor (NADPH/NADP ${ }^{+}$) turn-over rates in a CYP mediated reaction by pressure-assisted CE under reverse polarity. Samples were injected at the shortend of the fused-silica capillary with an $11 \mathrm{~cm}$ effective length for on-line UV absorbance detection (254 $\mathrm{nm})$. Reverse polarity allows anionic analytes to travel towards the detector, while a 0.1 psi forward pressure (counter to EOF) aids in optimizing resolution and analysis times due to the strong EOF under alkaline buffer conditions (100 mM borate, $\mathrm{pH}$ 9.2). In this case, $\mathrm{NADP}^{+}$and $\mathrm{NADPH}$ have larger negative electrophoretic mobilities $\left(\mu_{e p}\right)$ than the internal standard ( $m$-nitrophenol, $\left.m-\mathrm{NO}_{2} \mathrm{Ph}\right)$ due to their high anionic charge density that is further increased by dynamic borate complexation during electromigration. 


\section{Table S2. CE-UV detection assay parameters for NADPH and NADP ${ }^{+}$quantification}

\begin{tabular}{cccccc}
\hline CPR Cofactors & $\begin{array}{c}\text { Measured } \\
\boldsymbol{R M T}^{\boldsymbol{a}}\end{array}$ & $\begin{array}{c}\boldsymbol{L O D} \\
(\boldsymbol{\mu M}, \mathbf{S} / \mathbf{N}=3)\end{array}$ & $\begin{array}{c}\mathbf{L O Q} \\
(\boldsymbol{\mu M}, \\
\mathbf{S} / \mathbf{N}=\mathbf{1 0})\end{array}$ & $\begin{array}{c}\text { Linearity }^{b} \\
\left(\boldsymbol{R}^{2}\right)\end{array}$ & $\begin{array}{c}\text { Precision }^{c} \\
(\boldsymbol{\%} \boldsymbol{C V})\end{array}$ \\
\hline $\mathrm{NADP}^{+}$ & $2.1 \pm 0.1$ & 0.20 & 0.60 & 0.9999 & 3.2 \\
$\mathrm{NADPH}$ & $2.3 \pm 0.1$ & 0.25 & 0.70 & 0.9997 & 8.8 \\
\hline
\end{tabular}

a. $R M T=$ relative migration time

b. Calibration curves derived from linear regression of three inter-day triplicate runs $(n=9)$ over a 100-fold range from 0.25-25

$\mu M$ where measured responses were normalized to the internal standard, $m$-nitrophenol. $C V=$ cofactor of variance

c. Average reproducibility for cofactor quantification by $C E$ with $U V$ detection at $254 \mathrm{~nm}$ for calibration solutions. 


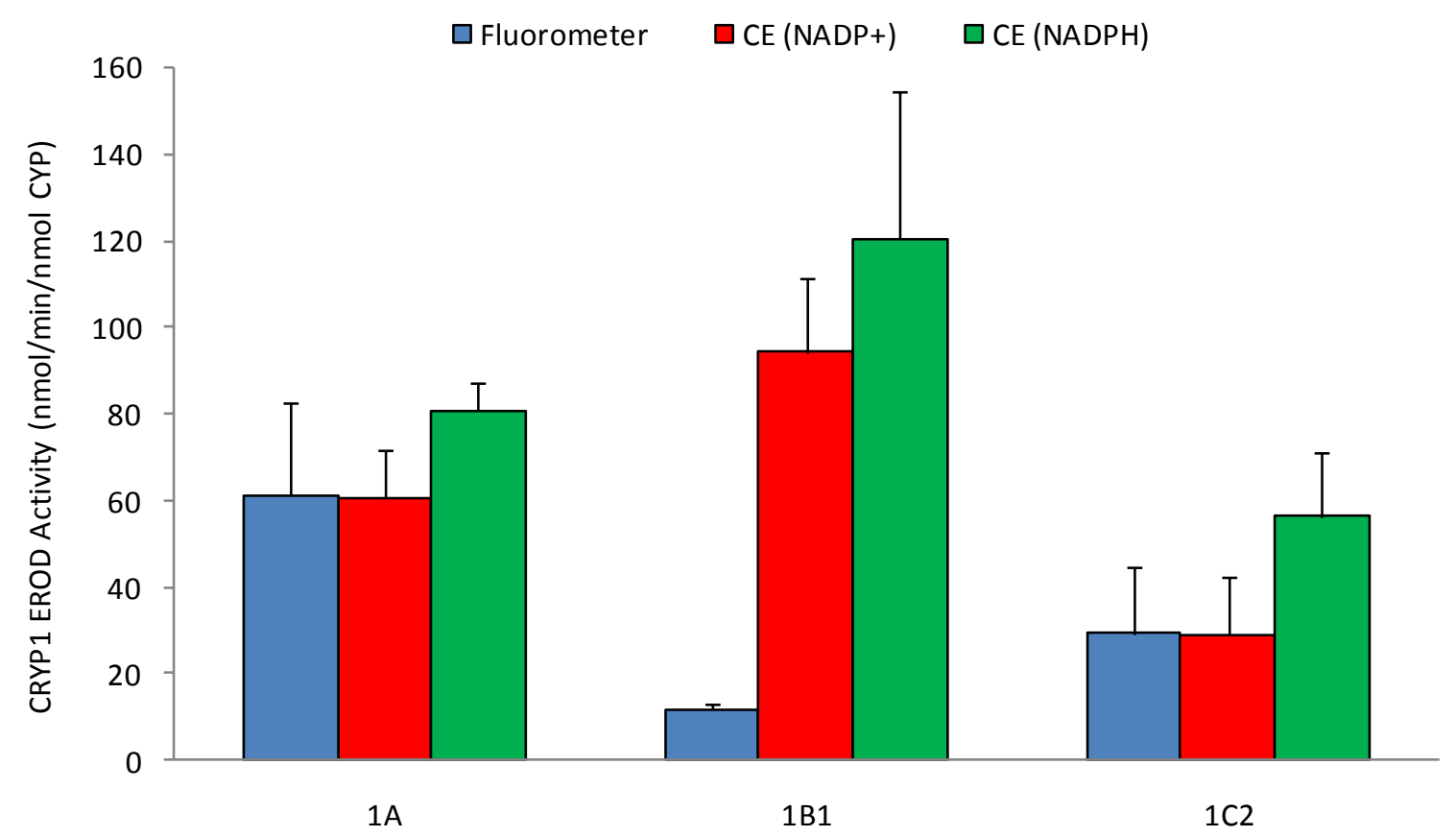

Figure S3. Comparison of zebrafish CYP1 activity for $O$-dealkylation of 7-ethoxyresorufin (ER) as a model fluorogenic substrate probe based on rates of NADPH and NADP ${ }^{+}$(by CE) relative to product turn-over (fluorescence). NADPH responses were found to be generally less accurate compared to $\mathrm{NADP}^{+}$for estimation of product turn-over rates for highly coupled CYP-catalyzed reactions involving ER, such as CYP1A and CYP1C2. 


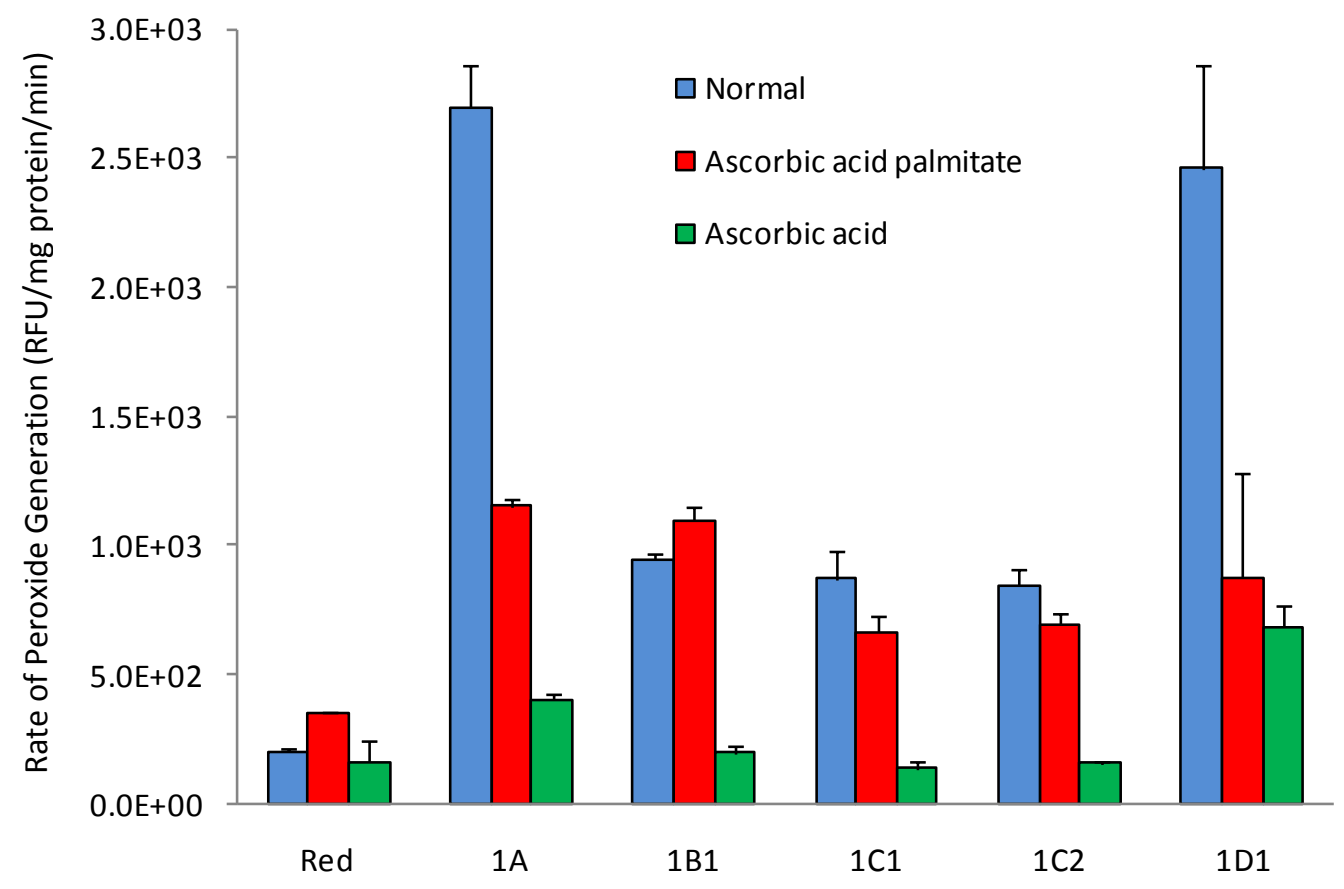

Figure S4. Impact of ascorbic acid palmitate (AAP) and ascorbic acid (AA) as hydrogen peroxide $\left(\mathrm{H}_{2} \mathrm{O}_{2}\right)$ scavengers in CYP1 mediated reactions with 17ß-estradiol (E2). AAP or AA (1 mM) were added to reactions for the fluorometric measurement of $\mathrm{H}_{2} \mathrm{O}_{2}$ production by dichlorofluorescein (DCF; 10 $\mu \mathrm{M}$ final reaction concentration) in CYP1 mediated reactions with E2 $(50 \mu \mathrm{M})$. Decreased rates of $\mathrm{H}_{2} \mathrm{O}_{2}$ were consistently measured when AAP and notably AA were present as a lipophilic and hydrophilic antioxidant, respectively. Note that the reductase blank (no CYP1) was found to have significantly higher rates of $\mathrm{H}_{2} \mathrm{O}_{2}$ production only with AAP, which was in all cases much lower than reactions with hemecontaining CYP1s. 


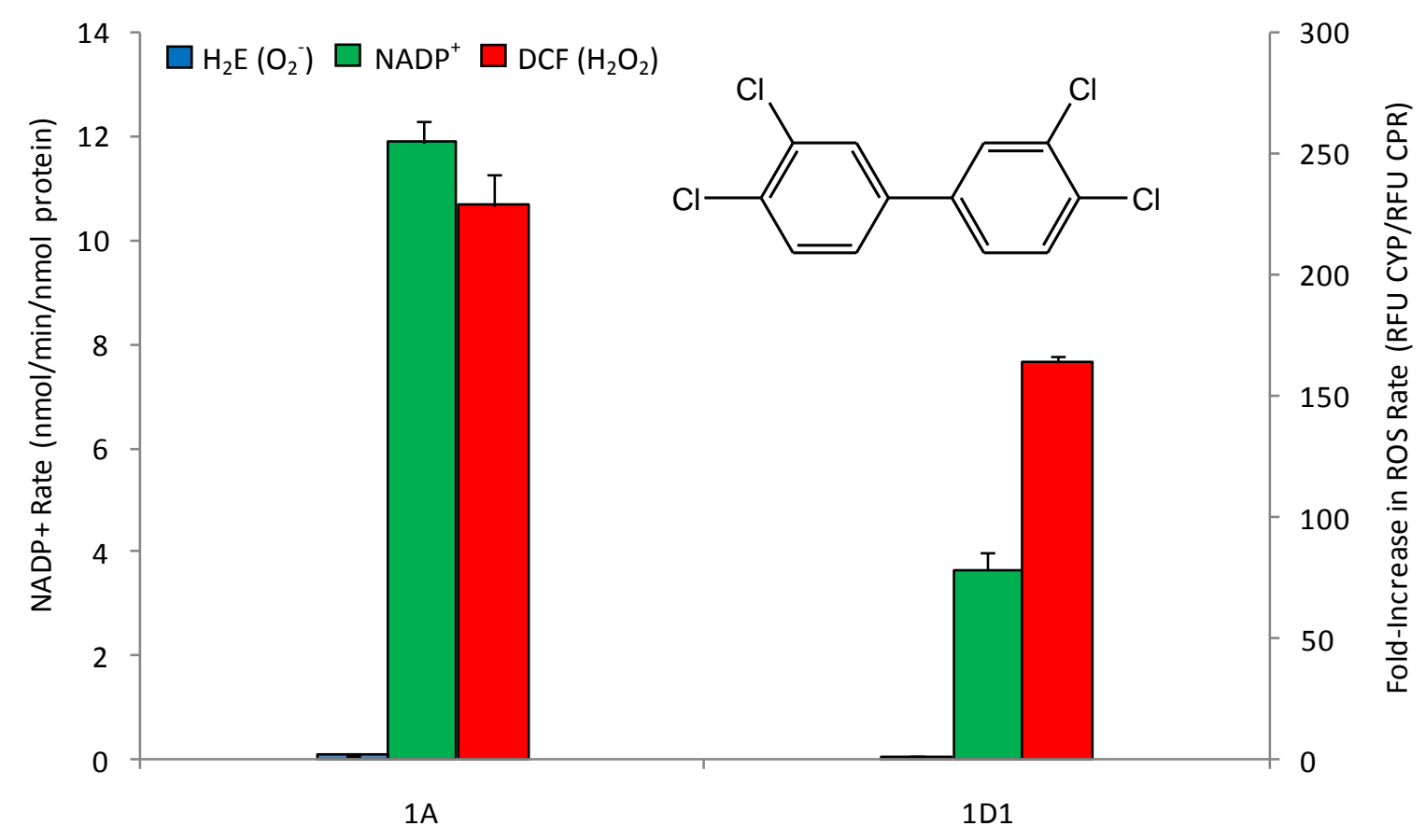

Figure S5. Comparison of extent of CYP1 uncoupling induced by PCB 77 as reflected by rates of $\mathrm{NADP}^{+}$production and responses of two fluorogenic ROS probes, dihydroethidium $\left(\mathrm{H}_{2} \mathrm{E}\right)$ and dichlorofluorescein for superoxide anion $\left(\mathrm{O}_{2}{ }^{-}\right)$and hydrogen peroxide $\left(\mathrm{H}_{2} \mathrm{O}_{2}\right)$, respectively. CYP1 isoforms were incubated in reaction buffer $(50 \mathrm{mM}$ tris, $0.1 \mathrm{M} \mathrm{NaCl}, \mathrm{pH} 7.8)$ together with $10 \mu \mathrm{M}$ PCB 77 and DCF or HE (10 $\mu \mathrm{M}$ final reaction concentration) prior to initiation of the enzymatic reaction with $1.4 \mathrm{mM}$ NADPH. Rates of $\mathrm{NADP}^{+}$production are in $\mathrm{nmol} \mathrm{NADP} / \mathrm{min} / \mathrm{nmol} \mathrm{CYP}$, whereas ROS response ratios were normalized to reductase blanks. Overall, $\mathrm{H}_{2} \mathrm{O}_{2}$ was the major ROS enhanced over 230 and 160-fold for CYP1A and CYP1D1, respectively when incubating with PCB 77 relative to only minor increases in $\mathrm{O}_{2}{ }^{-}$in comparison to background reductase blank experiments. Similar to previous enzymatic kinetic studies with E2 and fluorogenic substrate probes, CYP1A overall had greater catalytic activity relative to CYP1D1. 


\title{
Chapter 3 :
}

\section{Dichlorofluorescein product separation with CE-UV}

\begin{abstract}
:
Cytochrome P450s (CYPs) are functionally diverse monooxygenases that receive their reducing equivalents from the cofactor NADPH. The stoichiometry of cofactor consumption and product formation is not always 1:1, and excessive NADPH consumption can lead to reactive oxygen species (ROS) formation. Previous studies have monitored NADPH consumption with capillary electrophoresis as a measure of metabolism of CYP isoforms, however the rates of true metabolism based on product formation may not be determined without monitoring ROS production. This study describes the method development for CE-UV detection of the ROS probe dichlorodihydrofluorescein (DCF). Data showed that UV absorbance detection was not adequate for measuring DCF formation in uncoupled CYP reactions, where only peroxide concentrations over $100 \mu \mathrm{m}$ could be consistently measured.
\end{abstract}




\subsection{Introduction:}

Reactive oxygen species (ROS) are group of chemically reactive neutral or free radical molecules formed as a by-product of molecular oxygen metabolism. This group of molecules include hydrogen peroxide $\left(\mathrm{H}_{2} \mathrm{O}_{2}\right)$, superoxide anion $\left(\mathrm{O}_{2}{ }^{--}\right)$, and hydroxyl radical $(\cdot \mathrm{OH})$, and have long been of interest to biologists as many biological processes generate ROS under normal or pathological conditions. Elevated levels of ROS is often associated with oxidative stress and chronic disease, while playing an essential role in redox control of cell signalling (Groeger et al., 2009)

Cytochrome P450 (CYP) mediated reactions can produce ROS due to biomolecular interactions involving the heme centre and molecular oxygen. Inefficient use of the electrons donated from NADPH can cause formation of $\mathrm{H}_{2} \mathrm{O}_{2}$ and $\mathrm{O}_{2}{ }^{\cdot-}$, which is converted to $\mathrm{H}_{2} \mathrm{O}_{2}$ (Huang and Szklarz, 2010). While some CYPs that metabolize endogenous compounds are efficient in cofactor consumption, others are involved in xenobiotic metabolism and are more prone to uncoupling of the catalytic cycle, resulting in elevated ROS release. This is thought to occur because xenobiotic metabolising CYPs have active sites that are more flexible to allow for docking of multiple different substrates (Denisov et al., 2005). While there are many methods to measure ROS production in vivo or in cell free systems, 2,7-dichlorodihydrofluoroscein diacetate ( $\left.\mathrm{DCFH}_{2}-\mathrm{DA}\right)$ as a fluorescent probe has been one of the most widely used methods to measure $\mathrm{H}_{2} \mathrm{O}_{2}$ (Chen et al., 2010). DCFH - DA has been used to measure CYP uncoupling (Schlezinger et al., 2006), yet, to our knowledge, it has not been used to measure excessive cofactor consumption for correction of CYP rate measurement.. 
Despite its use for almost 50 years, many of the mechanisms for $\mathrm{H}_{2} \mathrm{O}_{2}$ measurement using $\mathrm{DCFH}_{2}$-DA remains unclear. $\mathrm{H}_{2} \mathrm{O}_{2}$ does not directly interact with $\mathrm{DCFH}_{2}$-DA. Two acetate groups need to be cleaved either by esterase or with base to produce $\mathrm{DCFH}_{2}$, the molecule sensitive to $\mathrm{H}_{2} \mathrm{O}_{2}$. An electron transfer from $\mathrm{DCFH}_{2}$ with $\mathrm{H}_{2} \mathrm{O}_{2}$ forms the fluorescent product DCF (Figure 3.1). Because DCF and its parent molecules have hydroxyl and carboxylic acid groups, alkaline buffer conditions will cause the molecule to have a negative charge (mobility) due to ionization. The conjugated aromatic moiety of DCF allow for ultra-sensitive fluorescence or UV absorbance detection when using CE.

In Chapter 2, a CE-UV method for measuring cofactor consumption had been developed. However, measurement and correction for ROS produced in the CYP reactions was performed as a separate fluorometric assay. The goal of this study was to develop a separation for detection of DCF using CE-UV with the same general separation conditions from the method described in Chapter 2. If successful, one CE-UV method would be able to monitor NADPH/NADP+ turnover rates during CYP1 mediated reactions while also measuring for $\mathrm{H}_{2} \mathrm{O}_{2}$ production, giving a single integrated microseparation format for determining CYP activity.

\subsection{Materials and Methods:}

\subsubsection{Preparation and separation of $\mathrm{DCFH}_{2}$ by CE}

2,7-dichlorodihydrofluorscein diacetate $\left(\mathrm{DCFH}_{2}\right.$-DA, $20 \mathrm{mM}$ stock; Sigma) was

dissolved in ethanol and stored in darkness at $-20^{\circ} \mathrm{C}$. Acetate cleavage was achieved with 1.5 
$\mathrm{mM} \mathrm{NaOH}$ at RT for 10 min, producing a $200 \mu \mathrm{M} \mathrm{DCFH}_{2}-\mathrm{DA}$ working solution (modified from LeBel et al., 1992). Samples for the standard curve had $10 \mu \mathrm{M}$ of the hydrolysate $\mathrm{DCFH}_{2}$ and were prepared in diluted reaction buffer ( $5 \mathrm{mM}$ tris $10 \mathrm{mM} \mathrm{NaCl}, \mathrm{pH} 7.8)$, with $1 \mu \mathrm{M}-1 \mathrm{mM}$ concentrations of $\mathrm{H}_{2} \mathrm{O}_{2}$ prepared fresh from a $30 \%$ solution.

\subsubsection{CYP1A Membrane preparation and CYP reactions}

Bacterial membranes with active zebrafish CYP1A were prepared as described in Chapter 2 and by Scornaienchi et al. (2010). Membranes were diluted to 70 nM CYP1A in membrane buffer (50 mM Tris-acetate, $0.25 \mathrm{mM}$ EDTA, $250 \mathrm{mM}$ sucrose, $\mathrm{pH}$ 7.6) and used for kinetic assays. Reactions involving CYP1A had $3 \mu \mathrm{M}$ 7-pentoxyresorufin (7PR) with $10 \mu \mathrm{M}$ $\mathrm{DCFH}_{2}$ made in reaction buffer (50 mM tris, $\left.0.1 \mathrm{M} \mathrm{NaCl}, \mathrm{pH} 7.8\right)$, were initiated with NADPH $(20 \mu \mathrm{M})$ and were incubated $28^{\circ} \mathrm{C}$ for 10 minutes. Reactions were quenched with ice-cold methanol in a 1:1 ratio. CYP1A with 7PR was chosen as it had a $2.4 \%$ coupling efficiency (product/cofactor $\mathrm{x} 100 \%$ ) with a large cofactor consumption (46 nmol NADP ${ }^{+} / \mathrm{nmol}$ CYP1A/min; Chapter 2), making it an ideal candidate to explore $\mathrm{H}_{2} \mathrm{O}_{2}$ production in a CYP mediated reaction.

\subsubsection{Separation of Samples by CE-UV}

Samples were kept on ice in the dark until transferred to a $4{ }^{\circ} \mathrm{C}$ sample tray. Samples were separated in a $40 \mathrm{~cm}$ long fused-silica capillary $(75 \mu \mathrm{m}$ inner diameter, $29 \mathrm{~cm}$ effective length to detector, $25^{\circ} \mathrm{C}$ ) on a Beckman-Coulter P/ACE MDQ CE. The background electrolyte (BGE) was $100 \mathrm{mM}$ borate buffer, $\mathrm{pH}$ 9.2. The capillary was rinsed for 2 min with $1 \mathrm{M} \mathrm{NaOH}$ 
and 4 min with BGE before each sample at 20 psi. Samples were injected into the capillary at 0.5 psi for $10 \mathrm{~s}$ and run through the capillary to the detector using a $10 \mathrm{kV}$ under normal polarity with UV detection at $254 \mathrm{~nm}$. Peak areas were determined from electropherograms using the 32 Karat Software (Beckman Coulter, Inc, CA). These separation conditions differ from the CE-UV method described in Chapter 2, as they had a normal polarity (as opposed to reverse polarity) with sample injection at the inlet, causing the separation path in the capillary to be $\sim 29 \mathrm{~cm}$, compared to $\sim 11 \mathrm{~cm}$ separation path described in Chapter 2 .

\subsection{Results and Discussion}

Initial CE-UV separations without protein, containing $\mathrm{DCFH}_{2}$ and $\mathrm{H}_{2} \mathrm{O}_{2}$ (concentration > $100 \mu \mathrm{M}$; incubated for $10 \mathrm{~min}, \mathrm{pH}$ 7.8) showed baseline resolution of $\mathrm{DCFH}_{2}-\mathrm{DA}, \mathrm{DCFH}_{2}$, DCF, and an unknown product that is predicted to be an open-ringed $\mathrm{DCFH}_{2}$ hydrolysate (Figure 3.2). $\mathrm{H}_{2} \mathrm{O}_{2}$ concentrations less than $100 \mu \mathrm{M}$ did not show a detectable peak with $\mathrm{UV}$ for DCF after a 15 min incubation period. A base concentration of $1.5 \mathrm{mM} \mathrm{NaOH}$ produced the maximal amount of $\mathrm{DCFH}_{2}$. Higher concentrations of base resulted in lower concentrations of $\mathrm{DCFH}_{2}-$ DA, however it formed excessive amounts of the unknown DCF-hydrolysis product. Similarly, lower concentrations of base formed less $\mathrm{DCFH}_{2}$. At $\mathrm{H}_{2} \mathrm{O}_{2}$ concentrations $>1 \mathrm{mM}$, large amounts of the DCF-hydrolysis product were formed along with measurable amounts of DCF.

DCF peak formation was confirmed by taking sample aliquots from reactions after CEUV analysis, and measuring fluorescence with a fluorimeter (excitation $488 \mathrm{~nm}$, emission 530 nm). Peak area measured by CE-UV was compared to fluorescence signal and showed a linear 
correlation $\left(R^{2}=0.99\right.$; data not shown) for $\mathrm{H}_{2} \mathrm{O}_{2}$ additions between $100 \mu \mathrm{M}-1 \mathrm{M}$. At concentrations lower than $100 \mu \mathrm{M} \mathrm{H}_{2} \mathrm{O}_{2}$, the formation of DCF was hard to quantify with CE$\mathrm{UV}$, however fluorescent signals were present when measured with a fluorimeter. $\mathrm{DCFH}_{2}$ appeared to undergo autooxidation over time, which may be due to the presence of tris buffer (Chen et al., 2010). The autooxidation to DCF prevented accurate measurement of $\mathrm{H}_{2} \mathrm{O}_{2}$ in stopped reactions for samples that were awaiting analysis for longer than 2 hours. This made it difficult to get a stable and repeatable measurement of $\mathrm{H}_{2} \mathrm{O}_{2}$ for samples which could not be analyzed immediately.

When $\mathrm{DCFH}_{2}$ was included in a reaction with CYP1A and 7PR, there was no quantifiable DCF formation for reactions with incubations of $10 \mathrm{~min}$ and 2 hours. CYP1A reactions with 7PR consumed large amounts of cofactor with little product formation, however, there was no measurable DCF formation detected with the CE-UV method. We were unable to confirm $\mathrm{H}_{2} \mathrm{O}_{2}$ production via fluorescence as spectral interference with resorufin (excitation/emission $488 \mathrm{~nm} / 530 \mathrm{~nm}$ ) overlapped with DCF (excitation/emission $488 \mathrm{~nm} / 530$ $\mathrm{nm}$ ), giving artefacts in the data. This suggests that CYP1A with 7PR reaction was either an uncoupled reaction that produced $\mathrm{H}_{2} \mathrm{O}_{2}$ which our CE-UV was unable to detect, or that there was no $\mathrm{H}_{2} \mathrm{O}_{2}$ produced. A fluorometric DCF assay was able to measure ROS production in uncoupled reactions with CYP1D1 with 17 $\beta$ - estradiol and CYP1A with PCB77 (Chapter 2), suggesting that the CE-UV method needs sensitivity improvement rather than the CYP1A/7PR reaction not forming ROS

The method can be improved both through instrumentation and through reaction conditions. To improve sensitivity in the DCF assay, measuring the product of $\mathrm{H}_{2} \mathrm{O}_{2}$ formation 
with a laser-induced fluorescence (LIF) detector coupled with the CE may give the sensitivity needed. A CE-LIF configuration with dual channel fluorescence detection would allow us to shorten the length of the separation similar to the $<3$ min separation described in Chapter 2 due to lack of spectral interferences since one channel measures the fluorescence of DCF while the second channel would measure the intrinsic fluorescence of NADPH. The potential for measuring NADPH through fluorescence (em $450 \mathrm{~nm}$ ) is feasible as there is no spectral interference with CYP heme that is separated by CE and/or denatured by methanol quenching (absorbs at $448 \mathrm{~nm}$ ). Overall, this work shows the potential to use CE separations in order to monitor NADPH cofactor concomitant to ROS production using DCF as a specific probe. With the flexibility, selectivity and sensitivity of LIF for simultaneous quantification of NADPH and/or DCF turn-over rates, this method would be effective as a single-step assay for screening of CYP function in terms of catalytic activity and coupling efficiency that corrects for excessive NADPH consumption due to uncoupling in CYP mediated reactions for any substrate or CYP. 


\section{References:}

Chen, X., Zhong, Z., Xu, Z., Chen, L. and Wang, Y. (2010). 2',7'-Dichlorodihydrofluorescein as a fluorescent probe for reactive oxygen species measurement: Forty years of application and controversy. Free Radic Res 44, 587-604.

Denisov, I. G., Makris, T. M., Sligar, S. G. and Schlichting, I. (2005). Structure and chemistry of cytochrome P450. Chem Rev 105, 2253-77.

Groeger, G., Quiney, C. and Cotter, T. G. (2009). Hydrogen peroxide as a cell-survival signaling molecule. Antioxid Redox Signal 11, 2655-71.

Huang, Q. and Szklarz, G. D. (2010). Significant increase in phenacetin oxidation on L382V substitution in human cytochrome P450 1A2. Drug Metab Dispos 38, 1039-45.

LeBel, C. P., Ischiropoulos, H. and Bondy, S. C. (1992). Evaluation of the probe 2',7'dichlorofluorescin as an indicator of reactive oxygen species formation and oxidative stress. Chem Res Toxicol 5, 227-31.

Schlezinger, J. J., Struntz, W. D., Goldstone, J. V. and Stegeman, J. J. (2006). Uncoupling of cytochrome P450 1A and stimulation of reactive oxygen species production by co-planar polychlorinated biphenyl congeners. Aquat Toxicol 77, 422-32.

Scornaienchi, M. L., Thornton, C., Willett, K. L. and Wilson, J. Y. (2010). Functional differences in the cytochrome P450 1 family enzymes from zebrafish (Danio rerio) using heterologously expressed proteins. Arch Biochem Biophys 502, 17-22. 

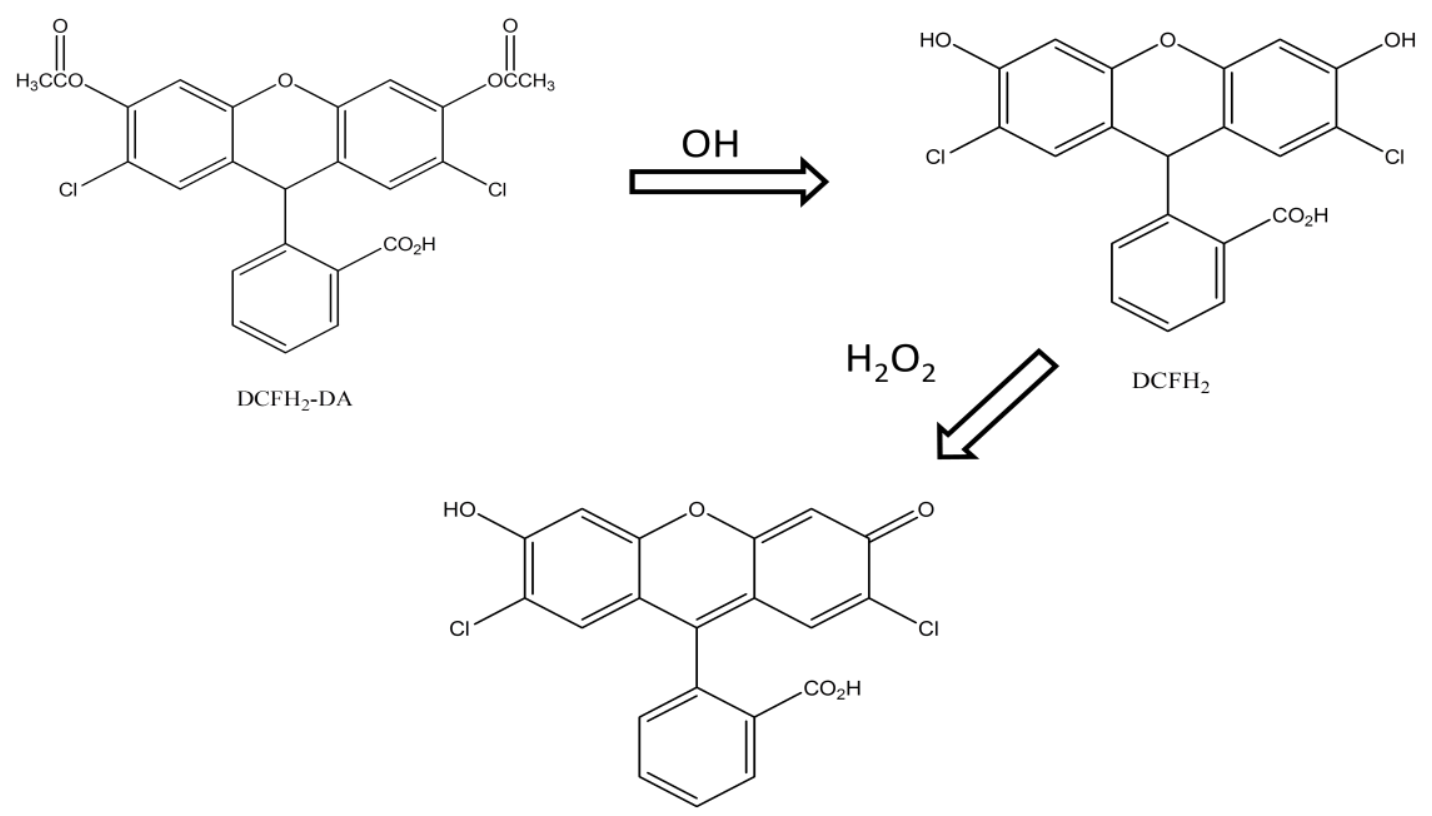

DCF

Figure 3.1: Reactions needed for the production of the fluorescent probe dichlorofluorescein. The initial 2,7-dichlorodihydrofluorescein diacetate $\left(\mathrm{DCFH}_{2}\right.$-DA) is exposed to base in order to cleave off the acetate groups, forming dichlorodihydrofluorescein $\left(\mathrm{DCFH}_{2}\right)$. $\mathrm{DCFH}_{2}$ is able to react with peroxide or other reactive oxygen species in order to form the fluorescent product dichlorofluorescein (DCF). 


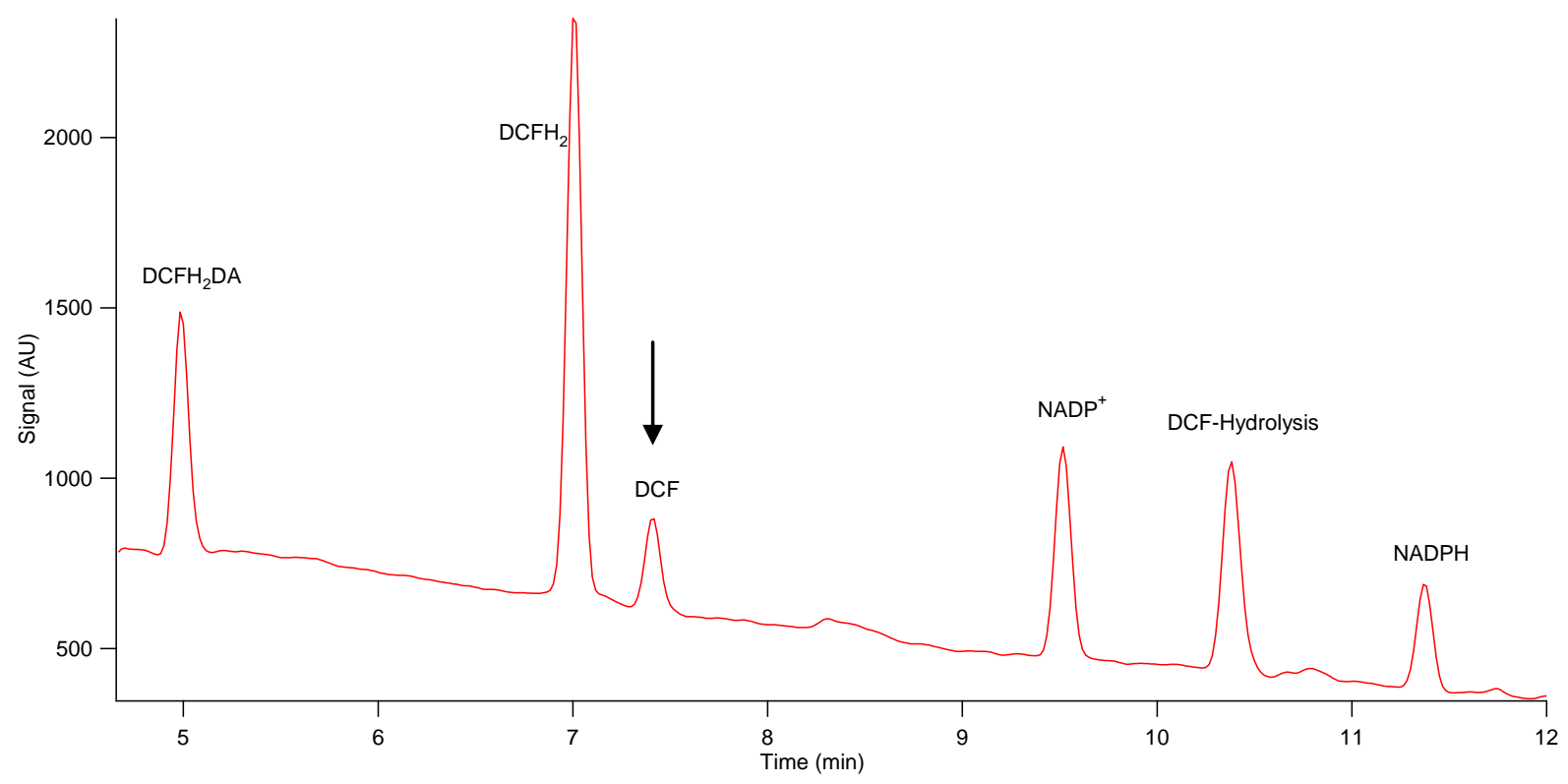

Figure 3.2: Separation of 2,7-dichlorodihydrofluorescein diacetate products with CE. 2,7-

dichlorodihydrofluorescein diacetate $\left(\mathrm{DCFH}_{2}-\mathrm{DA}\right)$ was exposed to $1.5 \mathrm{mM} \mathrm{NaOH}$ to cleave off acetyl groups, forming dichlorodihydrofluorescein $\left(\mathrm{DCFH}_{2}\right) .100 \mu \mathrm{M} \mathrm{H}_{2} \mathrm{O}_{2}$ was added to the solution and allowed to incubate for 15 min before separation, which reacted with the $\mathrm{DCFH}_{2}$ to form dichlorofluorescein (DCF). In addition to DCF formation, a DCF-hydrolysis product formed, potentially formed through the opening of the central ring. A $40 \mathrm{~cm}$ long fused-silica capillary $(75 \mathrm{uM}$ inner diameter, $29 \mathrm{~cm}$ length to detector, $25^{\circ} \mathrm{C}$ ) was used for separation on a Beckman-Coulter P/ACE MDQ CE. The background electrolyte (BGE) was $100 \mathrm{mM}$ borate buffer, $\mathrm{pH} 9.2$. 
MSc Thesis - J.G. Harskamp McMaster University - Biology 


\section{Chapter 4:}

\section{General Discussion}

In this study, a novel method for measuring cytochrome P450 family 1 (CYP1) activity with capillary electrophoresis was developed. The cofactor NADPH, the electron donor in CYP mediated reactions, was monitored as a means to measure CYP1 activity in reactions with five zebrafish (Danio rerio) CYP1s coexpressed with human cytochrome P450 reductase for six fluorometric substrates and 17 $\beta$-estradiol (E2). CYP1 activity from cofactor consumption was compared to fluorescent product formation. Separations showed cofactor consumption for a majority of synthetic fluorometric substrates and E2; 4 substrates in CYP reactions had greater than $80 \%$ coupling efficiency for synthetic substrates, and 2 CYPs showed greater than $90 \%$ coupling efficiency for E2 when measured by CE and GC-MS. Differences in product formation and cofactor consumption were found in the remaining substrate/CYP reactions with coupling efficiencies under 50\%, suggesting uncoupled reactions and the production of reactive oxygen species (ROS). ROS production was determined with the fluorescent probes dichlorofluorescein (DCF) and dihydroethidium (HE); DCF responses indicated that hydrogen peroxide was released in uncoupled reactions. To the best of our knowledge, this is the first study that has examined differential coupling efficiency of several CYP isoforms with various substrates.

\subsection{CE separation of CYP reactions}

CE proved to be an effective separation technique for the detection and quantification of CYP activity. Measuring CYP activity is important in pharmacology and toxicology, with the 
most well known application being drug development. With a handful of CYPs being responsible for metabolism of the majority of pharmaceuticals available (Guengerich, 2001), understanding how CYPs interact with a novel drug is imperative in pre-clinical trials. There has been an increase of studies investigating CYP metabolism with CE. A study investigating the chiral substrates and metabolites of CYP3A4 mediated verapamil metabolism took advantage of CE to separate enantiomers in the reaction (Ha et al., 2006). CYP3A4 metabolism of quinidine has used electrophoretically mediated microanalysis (EMMA) which treats the capillary as an online reactor before separation (Bhoopathy et al., 2001). EMMA has been used since the early 1990's and is a technique which benefits $\mathrm{CE}$ in its automation of enzyme reactions, further allowing $\mathrm{CE}$ to be used as a separation technique capable of high through-put screening (HTS; Bao et al., 1997). Only a handful of studies have investigated CE as a tool to quantify NADPH consumption in CYP mediated reactions to determine CYP activity (Zhang et al., 2008; Zhang et al., 2006).

For our purposes, CE was used to resolve the intrinsically charged cofactors NADPH and $\mathrm{NADP}^{+}$. By altering the numerous variables in a $\mathrm{CE}$ separation, such as buffer, buffer $\mathrm{pH}$, capillary length, voltages settings, sample injection length, and detection wavelength, a method was developed that could separate and measure NADPH and NADP+ in under 3 min from quenched CYP1 reactions. Initial difficulties arose with developing a short separation of the very similar NADPH and NADP ${ }^{+}$. Previous studies had CE separations of NADPH and NADP ${ }^{+}$ achieved in approximately 15 min (Zhang et al., 2006), however our end goal of a HTS method needed a much shorter separation. A counter pressure against the electroosmotic flow (EOF) was used to adjust the migration time needed for adequate resolution within a minimum analysis time. Yet, the separation was sensitive enough for detection of $\mathrm{NADP}^{+}$at $0.2 \mu \mathrm{M}$ (See Table S2 in Chapter 2). The UV detection at $254 \mathrm{~nm}$ reduced spectral interferences with low background 
noise for reliable quantification of NADPH and $\mathrm{NADP}^{+}$. An absence of tris buffer in the reaction caused an increase in noise of the signal, and any amount over $1 \mathrm{mM}$ in the sample greatly reduced the background noise and stabilized the signal. The methanol used to quench the reaction acted as the EOF marker in the separation. In general, the final conditions for the $\mathrm{CE}$ method allowed for a quick separation with little sample handling which can be readily translated to a high-throughput format when using a capillary array system.

\subsection{ROS detection and separation of DCF and HE by CE}

CYP mediated reactions can form $\mathrm{H}_{2} \mathrm{O}_{2}$ and superoxide from the uncoupling of the catalytic cycle in which electrons donated from NADPH form unstable intermediates (Gorsky et al., 1984). Techniques to measure superoxide and $\mathrm{H}_{2} \mathrm{O}_{2}$ have utilized colourmetric, fluorescence, and derivitization for both intact or cell free systems (Bohmer et al., 2011; Rhee et al., 2010; Xu et al.). Due to the sensitivity of fluorescent probes and the ability to measure ROS production in vivo, 2,7-dichlorofluorescein (DCF) and dihydroethidium (HE) have been a very popular choice

for measuring $\mathrm{H}_{2} \mathrm{O}_{2}$ and superoxide, respectively. However, even with their widespread use, critics have raised the question of the reliability of these probes, as multiple fluorescent products can be formed with HE, and common enzymes such as superoxide dismutase and catalase can interfere with DCF measurements. (Chen et al., 2010; Zhao et al., 2003). Superoxide is very labile and quickly transmutes to $\mathrm{H}_{2} \mathrm{O}_{2}$ via superoxide dismutase (SOD) and has the tendency to form more than one fluorescent product with HE when used in intracellular studies. (Zhao et al., 2003). This complicates analyses in cell free systems where separation techniques are not utilized, such that multiple fluorescent products of similar excitation/emission which are not 
directly associated with superoxide would give an inflated signal and would overestimate superoxide formation. Studies investigating superoxide formation have used separation techniques such as HPLC (Zielonka et al., 2009) and CE (Xu et al., 2010) in order to avoid the interference of additional fluorescent products, however many studies opt to simply measure $\mathrm{H}_{2} \mathrm{O}_{2}$ production assuming that much of the superoxide quickly forms $\mathrm{H}_{2} \mathrm{O}_{2}$ in a reaction catalyzed by SOD.

Our data confirmed the production of $\mathrm{H}_{2} \mathrm{O}_{2}$ in CYP1 mediated reactions with the use of DCF and HE, respectively. Superoxide was not detected in these reactions with the use of HE. CYP1D1 mediated E2 metabolism was uncoupled, as evidenced by large NADPH consumption and low product formation (Chapter 2). Significant $\mathrm{H}_{2} \mathrm{O}_{2}$ production was measured with DCF in CYP1D1 reactions with E2 (Chapter 3). Fluorescent detection of DCF in uncoupled reactions involving E2 and CYP1 isoforms were successful using a plate reader (Chapter 2), CE approaches were less successful (Chapter 3).

Initial attempts to detect $\mathrm{H}_{2} \mathrm{O}_{2}$ used a CE-UV separation measuring DCF formation in a CYP mediated reaction (Chapter 3), however, the method was unsuccessful because the sensitivity was too low. Only $100 \mu \mathrm{M} \mathrm{H}_{2} \mathrm{O}_{2}$ could be detected with CE-UV whereas the limit of detection with fluorescence detection was sub micromolar. The desire to use a CE separation of DCF products was based on two factors. First, HTS screening CYP activity requires co-detection of ROS and NADPH consumption in a single CE-based assay. Second, our use of fluorescent assays for CYP activity resulted in spectral overlap between resorufin (excitation $528 \mathrm{~nm}$, emission $590 \mathrm{~nm}$ ) and DCF (excitation $488 \mathrm{~nm}$, emission $530 \mathrm{~nm}$ ), complicating plate based assays of DCF with the resorufin substrates. Thus, measurement of both DCF and resorufin 
product formation by fluorimeter was difficult. Fluorescent detection of DCF was possible in CYP1 reactions with E2 and PCB77 using a plate reader assay, however. Reactions with E2, a non-fluorescent substrate which exhibited uncoupling for CYP1s, had measureable DCF formation when measured by a fluorimeter. This supports the notion that uncoupled reactions had significant ROS production (Chapter 2). PCB 77 was known to cause significant uncoupling with fish CYP1A (Schlezinger et al., 2006) and was used as a positive control. Reactions of CYP1A with PCB77 had large NADPH consumption (12 nmol nmol CYP $\left.{ }^{-1} \min ^{-1}\right)$, measured by CE, and high DCF formation, measured by fluorimeter, which confirmed that the rates of DCF formation in CYP mediated reactions were due to $\mathrm{H}_{2} \mathrm{O}_{2}$ formation. Rates of CYP1A mediated hydroxylation of PCB77 are quite low with rates under 1 pmol/min/nmol CYP (Prasad et al., 2007).

Additional efforts to merge ROS detection with the CE assays (see Chapter 2 and Chapter 3) would increase the usefulness of CE for detection of CYP activity. The two methods described for measuring cofactor consumption and DCF production, respectively, have similar separation conditions, except the cofactor separation uses a short-end injection and reverse polarity as a longer separation time was needed to resolve the different peaks from the DCF compounds. However, because the only analyte of significance in DCF separation had fluorescent properties, (Chapter 3), a shortened separation length (from $\sim 13 \mathrm{~min}$ to $<3 \mathrm{~min}$ ) would still yield the same fluorescent signal, making the methods compatible. By measuring NADPH with fluorescence (excitation/emission $340 \mathrm{~nm} / 450 \mathrm{~nm}$ ), a fluorescence detector on the CE would allow measurement of both DCF and NADPH. Furthermore, the potential to automate the reaction using electrophoretically mediated microanalysis (EMMA) would further increase automation and potential of a CE-based HTS assay. 


\subsection{Uncoupling of CYP reactions and inference of CYP function}

Coupling efficiency in CYP mediated reactions has been studied with most human drug metabolizing CYPs, however many isoforms still need to be investigated. In a study with human 1A2, five mutant CYP1A2 and wildtype enzyme were studied to see if catalytic rate and coupling efficiency were altered in their metabolism of phenacetin (Huang and Szklarz, 2010). The catalytic rate for the wild type enzyme was the highest but the coupling efficiency of the wildtype and the mutants was less than $3 \%$ (product formed/cofactor consumption x 100\%); coupling efficiency for CYP1A2 and a synthetic coumarin based substrate was under $8 \%$ (Traylor et al., 2011). Human CYP3A4, bioengineered with a Bacillus megaterium reductase domain showed coupling efficiency of less than $7 \%$ for all concentrations of the substrate erythromycin (Degregorio et al., 2011). The CYP1 reactions tested appeared to be inefficient in cofactor consumption for the majority of substrates. CYP isoforms responsible for xenobiotic metabolism are predicted to have lower coupling efficiencies because their active sites accommodate multiple substrates and often produce multiple metabolites per substrate (Denisov et al., 2005).

The most coupled reaction appeared to be CYP1A with 7-ethoxyresorufin (7-ER), which is encouraging as it is the preferred method for determining CYP1A1 activity in mammals (Ansede and Thakker, 2004) and CYP1A activity in fish (Wang et al., 2008). While 7-ER is metabolized by all CYP1s except CYP1D1 (Scornaienchi et al., 2010), rates of 7-ER metabolism are considered indicative of CYP1A protein levels and EROD is a widely used biomarker in fish exposed to environmental toxins (Lee and Anderson, 2005) or in studies of CYP induction 
(Smith and Wilson, 2010). The high coupling efficiency appears to solidify the use of this probe and an effective means for identifying CYP1A activity. Although zebrafish CYP1C1 and 1C2 metabolized the common alkoxyresorufin substrates, the majority of reactions were uncoupled.

CYP1D1, an isoform with unknown function, showed uncoupling for all the synthetic fluorometric substrates (except CEC) and for the endogenous hormone E2. This is not surprising since catalytic rates of CYP1D1 with these substrates were low. CYP1D1 mediated benzo[a]pyrene metabolism was moderate suggesting the CYP1D1 active site can accommodate planar, aromatic substrates (Scornaienchi et al., 2010). While there was no evidence of significant metabolite formation with CYP1D1 and the substrates tested, that the substrates were able fit into the CYP1D1 active site was indicated by NADPH consumption in the reactions. The evolutionary similarity of CYP1A and CYP1D1 in zebrafish has been discussed previously (Goldstone et al., 2009), and is functionally confirmed by the similarity of substrates that can induce cofactor consumption. It would not be surprising if CYP1A could metabolise endogenous or preferred CYP1D1 substrates. The expression patterns are quite different for CYP1A and CYP1D1. CYP1D1 is more highly expressed in early development, suggesting that CYP1A and CYP1D1 have different physiological roles (Goldstone et al., 2009). While caffeine, a mammalian CYP1A2 substrate, was not tested as a substrate for zebrafish CYP1D1, cynomolgus monkey CYP1D1 showed low rates of caffeine metabolism (less than 50 pmol nmol P450 ${ }^{-1} \mathrm{~min}^{-}$

$\left.{ }^{1}\right)$ suggesting another potential substrate in which to investigate uncoupling with CYP1D1.

\subsection{Oxidative stress in fish}

Xenobiotics, including polychlorinated biphenyls (PCBs) and polycyclic aromatic hydrocarbons (PAHs), have been shown to induce oxidative stress in aquatic organisms. These 
compounds are inducers and substrates of CYP1s and the oxidative stress is thought to be CYP mediated (Billiard et al., 2008; Schlezinger et al., 2006). Toxic metabolites may form from PCBs and PAHs; these metabolites may have a causal role in producing ROS. Our data has shown uncoupling for majority of the zebrafish CYP1 reactions tested, suggesting a direct oxidative stress from the CYP, independent from the metabolite product. In a study involving scup CYP1A, such high levels of ROS was produced in CYP1A reactions with 3,3,4,4tetrachlorobiphenyl (PCB 77) that the protein was inactivated and unable to metabolise 7ethoxyresorufin (Schlezinger et al., 2006). Likewise, in reactions run with zebrafish CYP1D1 with PCB 77, cofactor consumption stopped after 10 minutes during incubation, suggesting it became denatured and further suggesting direct oxidative stress to the protein.

\subsection{Future work}

While the method described in Chapter 2 was able to measure CYP1 activity and uncoupling, the potential of a single assay with little sample handing would allow for increased sample throughput and quantitation of CYP activity with any substrate. With the flexibility of $\mathrm{CE}$ as a separation technique, there is potential to use a variety of techniques for measuring CYP activity while keeping analysis time and cost low. The initial steps for combining ROS and $\mathrm{NADP}^{+}$measurements in a single CE assay would be to find a stable ROS probe. A selective probe for $\mathrm{H}_{2} \mathrm{O}_{2}$ would be preferable, as superoxide is very labile and forms $\mathrm{H}_{2} \mathrm{O}_{2}$ when transformed by superoxide dismutase. The use of a fluorimeter to measure DCF production to qualify $\mathrm{H}_{2} \mathrm{O}_{2}$ production was effective however the difficulties of measuring DCF in a CE-UV separation proved challenging (See Chapter 3). In our experiments, DCF autoxidised when 
samples sat on ice for over an hour, to the extent that the data was not consistent or repeatable; stability of DCF prior to detection is a challenge for the use of DCF as a ROS probe. The fluorescent properties of this probe could be utilized with a proper laser-induced fluorescence (LIF) coupled with preconcentration and an EMMA method, such that detection would be sensitive, and analysis could be performed directly in-capillary upon product formation, decreasing error due sample handling of labile ROS probes. The longer separation times for DCF described in Chapter 3 could also be shortened, or selective detection of oxidized DCF product, potentially allowing for a dual ROS/CYP assay with the $<3$ min separation described in Chapter 2. The merger of methods is possible as the majority of CE conditions are the same for each method, except for the short-end injection and reverse polarity of the configuration. This would give a CE-LIF method sensitive enough for DCF with a fast separation time.

Colorimetric/UV methods for detection of $\mathrm{H}_{2} \mathrm{O}_{2}$ have been described (Rhee et al., 2010). With the use of horseradish peroxidase (Degregorio et al., 2011) and/or ferrous catalysts (Huang and Szklarz, 2010), $\mathrm{H}_{2} \mathrm{O}_{2}$ can form a coloured product. While maximal absorbance may be at higher wavelengths, the conjugated or ring structure of some of the coloured products (e.g. xylenol orange or thiocyanate) will absorb at low UV detection allowing for probes which can be separated and measured in a CE-UV mediated reaction. The quenching and dilution step of the CYP mediated reaction provides a flexible time for derivatization, allowing for integration of compounds and incubations within the existing reaction method. In particular, a UV or fluorescent method for measuring $\mathrm{H}_{2} \mathrm{O}_{2}$ production utilizing the antioxidant qualities of reduced glutathione (GSH) and derivitization using $o$-phthalaldehyde (OPA) could provide the sensitivity needed (Bohmer et al., 2011; Kand'ar et al., 2007). $\mathrm{H}_{2} \mathrm{O}_{2}$ would react with GSH to form its oxidized dimer, GSSG. A derivatization step involving OPA, a benzene based dialdehyde, 
would form a stable derivative of GSH and add UV absorbing and fluorometric properties to the glutathione molecule (Bohmer et al., 2011). Reactions would have high concentrations of GSH; $\mathrm{H}_{2} \mathrm{O}_{2}$ would react with a portion of the GSH to form GSSG and the remaining GSH would react with OPA to form GS-OPA. A decrease in GS-OPA in the reaction would quantify ROS formation. The derivatization of GSH would be integrated in the quenching step and stabilize the products until they were detected with CE, unlike the unstable DCF product that needed to be sampled soon after the reaction.

To increase throughput, electrophoretically mediated microanalysis (EMMA) techniques could be employed. With the flexibility of CE as a separation platform, EMMA seeks to utilize the capillary as a reactor, allowing for online reactions to occur in capillary followed by a separation of reactants and metabolites. These on-line reactions greatly increase the automation available for the analysis, while decreasing the cost of the reaction as injection volumes may be reduced to $\mathrm{nL}$ (Fan and Scriba, 2010). The increased automation allows for the potential of high through-put screening, which would be useful for determining CYP function and screening novel compounds as CYP substrates.

A large number of novel CYPs have been identified and many proteins need to be characterized for function. While key isoforms in the mammalian CYP catalogue are well known and extensively researched, there is a need to investigate non-mammilian CYP function as they can differ from similar isoforms in mammals (Scornaienchi et al, 2010). Even in the well studied CYP1 family, new CYP1 subfamilies in non-mammals (sea urchin, tunicates, birds) have been discovered with no known function (Goldstone et al., 2007). Even more, the new CYP1D subfamily has been discovered in more mammilian species (Kawai et al., 2010), and still does 
not have in depth functional knowledge for cynomolgus monkey (Uno et al., 2011) and zebrafish (Scornaienchi et al., 2010). With access to a large chemical library and expressed proteins, small amounts of reactants could provide a cost effective way to screen a large number of different substrates to identify CYP function for novel isoforms. The coupling efficiency measured for these compounds would provide us functional information as to what type of structure can access the CYP active site. As an example, zebrafish CYP1D1 had a large uncoupling for aromatic planar molecules, most noteable being $\mathrm{E}_{2}$. We could then select a group of aromatic planar molecules to test for metabolism in order to decifer function. Additionally, in silico molecular modeling as demonstrated by Prasad et al. (2007), would be useful for further understanding how particular substrates enter into the CYP active site to elucidate further function. However, an initial single method capable of giving qualitative and quantitative measures of CYP activity could greatly improve the speed at which significant CYP/substrate combinations could be identified.

Our work showed the ability of a CE-UV method to quickly identify cofactor consumption in CYP1 reactions. This method was able to uncover the large discrepancy seen in cofactor usage by well established and relatively unknown CYP1s for fluorometric substrates that are extensively used. We were able to show that CYP1s displayed a range of coupling efficiencies for both xenobiotic and endogenous substrates, with most notably the large uncoupling of CYP1D1 with estradiol, giving hints as to which substrates the isoform is able to metabolize. With this assay, any CYP from any species can be tested for cofactor consumption for any substrate that does not co-migrate with $\mathrm{NADP}^{+}$or $\mathrm{NADPH}^{+}$, giving a strong tool for determining function for novel CYPs and for the study of CYP mediated ROS production through uncoupled reactions. 


\section{References:}

Ansede, J. H. and Thakker, D. R. (2004). High-throughput screening for stability and inhibitory activity of compounds toward cytochrome P450-mediated metabolism. J Pharm Sci 93, 239-55.

Bao, J. J., Fujima, J. M. and Danielson, N. D. (1997). Determination of minute enzymatic activities by means of capillary electrophoretic techniques. J Chromatogr B Biomed Sci Appl 699, 481-97.

Bhoopathy, S., Sarkar, M. and Thomas Karnes, H. (2001). A direct injection capillary electrophoretic technique for miniaturized high-throughput metabolic screening of the CYP 3A4 enzyme using quinidine as a probe. J Pharm Biomed Anal 25, 721-9.

Billiard, S. M., Meyer, J. N., Wassenberg, D. M., Hodson, P. V. and Di Giulio, R. T. (2008). Nonadditive effects of PAHs on Early Vertebrate Development: mechanisms and implications for risk assessment. Toxicol Sci 105, 5-23.

Bohmer, A., Jordan, J. and Tsikas, D. (2011). High-performance liquid chromatography ultraviolet assay for human erythrocytic catalase activity by measuring glutathione as o-phthalaldehyde derivative. Anal Biochem 410, 296-303.

Chen, X., Zhong, Z., Xu, Z., Chen, L. and Wang, Y. (2010). 2',7'-Dichlorodihydrofluorescein as a fluorescent probe for reactive oxygen species measurement: Forty years of application and controversy. Free Radic Res 44, 587-604.

Degregorio, D., Sadeghi, S. J., Di Nardo, G., Gilardi, G. and Solinas, S. P. (2011). Understanding uncoupling in the multiredox centre P450 3A4-BMR model system. J Biol Inorg Chem 16, 109-16.

Denisov, I. G., Makris, T. M., Sligar, S. G. and Schlichting, I. (2005). Structure and chemistry of cytochrome P450. Chem Rev 105, 2253-77.

Fan, Y. and Scriba, G. K. (2010). Advances in-capillary electrophoretic enzyme assays. $J$ Pharm Biomed Anal 53, 1076-90.

Goldstone, J. V., Jonsson, M. E., Behrendt, L., Woodin, B. R., Jenny, M. J., Nelson, D. R. and Stegeman, J. J. (2009). Cytochrome P450 1D1: a novel CYP1A-related gene that is not transcriptionally activated by PCB126 or TCDD. Arch Biochem Biophys 482, 7-16.

Gorsky, L. D., Koop, D. R. and Coon, M. J. (1984). On the stoichiometry of the oxidase and monooxygenase reactions catalyzed by liver microsomal cytochrome P-450. Products of oxygen reduction. J Biol Chem 259, 6812-7.

Guengerich, F. P. (2001). Common and uncommon cytochrome P450 reactions related to metabolism and chemical toxicity. Chem Res Toxicol 14, 611-50.

Ha, P. T., Sluyts, I., Van Dyck, S., Zhang, J., Gilissen, R. A., Hoogmartens, J. and VanSchepdael, A. (2006). Chiral capillary electrophoretic analysis of verapamil metabolism by cytochrome P450 3A4. J Chromatogr A 1120, 94-101.

Huang, Q. and Szklarz, G. D. (2010). Significant increase in phenacetin oxidation on L382V substitution in human cytochrome P450 1A2. Drug Metab Dispos 38, 1039-45.

Kand'ar, R., Zakova, P., Lotkova, H., Kucera, O. and Cervinkova, Z. (2007). Determination of reduced and oxidized glutathione in biological samples using liquid chromatography with fluorimetric detection. J Pharm Biomed Anal 43, 1382-7.

Lee, R. F. and Anderson, J. W. (2005). Significance of cytochrome P450 system responses and levels of bile fluorescent aromatic compounds in marine wildlife following oil spills. Mar Pollut Bull 50, 705-23.

Rhee, S. G., Chang, T. S., Jeong, W. and Kang, D. (2010). Methods for detection and measurement of hydrogen peroxide inside and outside of cells. Mol Cells 29, 539-49. 
Schlezinger, J. J., Struntz, W. D., Goldstone, J. V. and Stegeman, J. J. (2006). Uncoupling of cytochrome P450 1A and stimulation of reactive oxygen species production by co-planar polychlorinated biphenyl congeners. Aquat Toxicol 77, 422-32.

Scornaienchi, M. L., Thornton, C., Willett, K. L. and Wilson, J. Y. Functional differences in the cytochrome P450 1 family enzymes from zebrafish (Danio rerio) using heterologously expressed proteins. Arch Biochem Biophys 502, 17-22.

Scornaienchi, M. L., Thornton, C., Willett, K. L. and Wilson, J. Y. (2010). Functional differences in the cytochrome P450 1 family enzymes from zebrafish (Danio rerio) using heterologously expressed proteins. Arch Biochem Biophys 502, 17-22.

Smith, E. M. and Wilson, J. Y. (2010). Assessment of cytochrome P450 fluorometric substrates with rainbow trout and killifish exposed to dexamethasone, pregnenolone-16alpha-carbonitrile, rifampicin, and beta-naphthoflavone. Aquat Toxicol 97, 324-33.

Traylor, M. J., Chai, J. and Clark, D. S. (2011). Simultaneous measurement of CYP1A2 activity, regioselectivity, and coupling: Implications for environmental sensitivity of enzyme-substrate binding. Arch Biochem Biophys 505, 186-93.

Wang, Y., Zheng, R., Zuo, Z., Chen, Y. and Wang, C. (2008). Relation of hepatic EROD activity and cytochrome P4501A level in Sebastiscus marmoratus exposed to benzo[a]pyrene. J Environ Sci (China) 20, 101-4.

Xu, X., Thompson, L. V., Navratil, M. and Arriaga, E. A. Analysis of superoxide production in single skeletal muscle fibers. Anal Chem 82, 4570-6.

Zhang, J., Hoogmartens, J. and Van Schepdael, A. (2008). Kinetic study of cytochrome P450 by capillary electrophoretically mediated microanalysis. Electrophoresis 29, 3694-700.

Zhang, J., Lou, Y., Hoogmartens, J. and Van Schepdael, A. (2006). Screening of drug metabolism by CE. Electrophoresis 27, 4827-35.

Zhao, H., Kalivendi, S., Zhang, H., Joseph, J., Nithipatikom, K., Vasquez-Vivar, J. and Kalyanaraman, B. (2003). Superoxide reacts with hydroethidine but forms a fluorescent product that is distinctly different from ethidium: potential implications in intracellular fluorescence detection of superoxide. Free Radic Biol Med 34, 1359-68.

Zielonka, J., Hardy, M. and Kalyanaraman, B. (2009). HPLC study of oxidation products of hydroethidine in chemical and biological systems: ramifications in superoxide measurements. Free Radic Biol Med 46, 329-38. 